MATHEMATICS OF COMPUTATION

Volume 68, Number 228, Pages 1397-1427

S 0025-5718(99)01175-8

Article electronically published on May 25, 1999

\title{
OPTIMAL CONVERGENCE FOR THE FINITE ELEMENT METHOD IN CAMPANATO SPACES
}

\author{
GEORG DOLZMANN
}

\begin{abstract}
We prove a priori estimates and optimal error estimates for linear finite element approximations of elliptic systems in divergence form with continuous coefficients in Campanato spaces. The proofs rely on discrete analogues of the Campanato inequalities for the solution of the system, which locally measure the decay of the energy. As an application of our results we derive $W^{1, p_{-}}$-estimates and give a new proof of the well-known $W^{1, \infty_{-}}$-results of Rannacher and Scott.
\end{abstract}

\section{INTRODUCTION}

In this paper, we present a new approach to a priori estimates and error estimates for finite element solutions of linear elliptic systems of second order with continuous coefficients. Our results rely on an extension of the by now classical Campanato space methods in elliptic theory, which provide a powerful tool to prove regularity based on $L^{2}$ estimates rather than on an investigation of the fundamental solution. Estimates in the energy norm follow naturally from the variational structure of the problem.

We consider the elliptic system

$$
-\operatorname{div}(A D u)=-\operatorname{div} F \quad \text { in } \Omega,
$$

where $u \in W_{0}^{1,2}\left(\Omega ; \mathbb{R}^{m}\right)$ and $A$ satisfies the Legendre-Hadamard condition (see Sections 2 and 3 for the notation used in the introduction). Assume that $u_{h} \in$ $S_{0}^{h}\left(\Omega_{h}\right)$ is a solution of the corresponding weak formulation

$$
a\left(u_{h}, \psi_{h}\right)=\int_{\Omega} F D \psi_{h} d x \quad \forall \psi_{h} \in S_{0}^{h}\left(\Omega_{h}\right),
$$

where $S_{0}^{h}\left(\Omega_{h}\right)$ is the space of piecewise affine and globally continuous functions on a quasiuniform triangulation $\Omega_{h}$ of $\Omega$ and $a(\cdot, \cdot)$ is the bilinear form associated with $A$. Our first result concerns a priori estimates for $D u_{h}$ in Morrey and Campanato spaces. In particular we prove the following bound on $D u_{h}$ in the Campanato space $\mathcal{L}^{2, n}$ which is isomorphic to the space of functions of bounded mean oscillation studied in [JN]:

Received by the editor September 26, 1994 and, in revised form, October 2, 1997.

1991 Mathematics Subject Classification. Primary 65N12; Secondary 65N15, 65N30.

Key words and phrases. Optimal error estimates, finite element methods, Campanato spaces.

Partially supported by the Center for Nonlinear Analysis at Carnegie Mellon University, Pittsburgh and by Human Capital and Mobility, contract number ERBCHBGCT920004 at the University of Rome "La Sapienza".

(C)1999 American Mathematical Society 
Theorem 1.1. Assume that $\Omega$ is smooth, $A \in C^{0, \sigma}$ for some $\sigma>0$, and $F \in$ $\mathcal{L}^{2, n}(\Omega)$. Then $D u_{h} \in \mathcal{L}^{2, n}(\Omega)$, and we have the a priori estimate

$$
\left\|D u_{h}\right\|_{\mathcal{L}^{2, n}\left(\Omega_{h}\right)} \leq c_{n}\left(\left\|u_{h}\right\|_{2 ; \Omega_{h}}+\|F\|_{\mathcal{L}^{2, n}\left(\Omega_{h}\right)}\right) .
$$

The second main result is the following error estimate for the gradient of the finite element solution $u_{h}$.

Theorem 1.2. Assume that $\Omega$ is smooth, $A \in C^{0, \sigma}$, and the system (1.1) has a unique solution. Let $F \in \mathcal{L}^{2, n}(\Omega)$ and define $e_{h}=u-u_{h}$. Then

$$
\left\|D e_{h}\right\|_{\mathcal{L}^{2, n}\left(\Omega_{h}\right)} \leq c_{n} \inf _{w_{h} \in S_{0}^{h}}\left\|D u-D w_{h}\right\|_{\mathcal{L}^{2, n}\left(\Omega_{h}\right)} .
$$

The importance of estimates in $\mathcal{L}^{2, n}$ arises from the fact that this space is a natural substitute for $L^{\infty}$ in many results in real analysis. For example, if the system has a unique solution, Stampacchia's interpolation theorem [St] immediately implies the following $W^{1, p}$ estimate:

Theorem 1.3. Assume that $\Omega$ is smooth, $A \in C^{0, \sigma}$ for some $\sigma>0$, and the system (1.1) has a unique solution. Let $F \in L^{p}(\Omega)$ with $p \in(2, \infty)$. Then $D u_{h} \in L^{p}(\Omega)$, and we have the a priori estimate

$$
\left\|D u_{h}\right\|_{L^{p}\left(\Omega_{h}\right)} \leq c_{p}\|F\|_{L^{p}\left(\Omega_{h}\right)}
$$

as well as the error estimate

$$
\left\|D e_{h}\right\|_{L^{p}\left(\Omega_{h}\right)} \leq c_{p} \inf _{w_{h} \in S_{0}^{h}}\left\|D u-D w_{h}\right\|_{L^{p}\left(\Omega_{h}\right)} .
$$

As a further application of the $\mathcal{L}^{2, n}$-estimates we show in Section 7 how one can obtain optimal $W^{1, \infty}$-estimates for $e_{h}$, thus generalizing the famous result by Rannacher and Scott and the recent results in [SW2] to systems. This approach allows one to obtain uniform estimates by exploiting the variational structure of the problem, and does not rely on the weighted norm techniques first developed in $[\mathrm{Na}]$.

There exists an extensive literature on error estimates for finite element methods in various spaces. The question of whether optimal convergence holds in $W^{1, \infty}$ has been open for a long time and was finally solved by Rannacher and Scott in [RS]. Blum, Lin and Rannacher [BLR] showed in addition that in general the error $u-u_{h}$ is not of order $\mathcal{O}\left(h^{2}\right)$ in $L^{\infty}$ even if the data are smooth. The spaces $\mathcal{L}^{2, n}$ were used in $[\mathrm{R}]$ to prove optimal estimates for $D e_{h}$ up to a logarithmic factor, and in [Du2] to show optimal convergence for $e_{h}$ of order $\mathcal{O}\left(h^{2}\right)$ in two dimensions. General results in Orlicz spaces can be found in [Du1]. Schauder estimates for higher order methods have been analyzed in [Ni], while a discussion of properties of solutions of elliptic equations based on DeGiorgi's ideas has been carried out in [AC].

The paper is organized as follows. In Sections 2 and 3 we introduce our notation and summarize the basic results needed in the subsequent sections. We derive an analogue of the Campanato inequalities for the finite element solution in the interior situation in Section 4, while the boundary situation is analyzed in Section 5 . These estimates allow us to obtain the a priori estimates and the error estimates in Section 6, and uniform estimates are given in Section 7. Finally, the Appendix gives the proofs of some well-known results in elliptic theory.

While carrying out this programme, we shall state explicitly the necessary assumptions on the coefficients and the domain $\Omega \subset \mathbb{R}^{n}$ which ensure that the solution 
has the required regularity; that regularity theory for elliptic systems is more subtle than for elliptic equations can already be seen from the fact that there is no analogue of DeGiorgi's famous $C^{0, \sigma}$ regularity result for equations with $L^{\infty}$ coefficients. In addition, Gårding's inequality does not hold for $L^{\infty}$ coefficients, see [Zh]. The approach towards regularity pursued here is unfortunately based on Hölder continuity of the coefficients. We therefore do not recover the general estimates in [BS] for equations in the scalar case.

\section{Preliminaries}

Let $\Omega \subset \mathbb{R}^{n}$ be a convex, open and bounded domain and define $\Omega\left(x_{0}, R\right)=$ $B\left(x_{0}, R\right) \cap \Omega$. The convexity assumption is not related to regulartity properties of the solution (in the scalar case it implies the square integrability of the second derivatives); it only avoids extending the coefficients outside of $\Omega$. For methods to treat nonconvex domains, see e.g. [SW1]. We say that $\Omega$ is a domain of class $C^{k, \sigma}$ if for all $x_{0} \in \partial \Omega$ there exists a diffeomorphism $\gamma \in C^{k, \sigma}\left(B_{R}^{+} ; \mathbb{R}^{n}\right)$ which maps $B_{R}^{+}$onto $\Omega\left(x_{0}, R\right)$ and $\Gamma_{R}$ onto $\partial \Omega\left(x_{0}, R\right) \cap \partial \Omega$. Here $B_{R}^{+}=\left\{x \in \mathbb{R}^{n}\right.$ : $\left.|x|<R, x_{n}>0\right\}$ and $\Gamma_{R}=\left\{x \in \mathbb{R}^{n}:|x|<R, x_{n}=0\right\}$. We say that $\mathcal{T}_{h}$ is a quasiuniform triangulation of $\Omega$ with $n$-simplices $T$ if there exist constants $\sigma_{0}$, $\sigma_{1}>0$ independent of $h$ such that for each $T \in \mathcal{T}_{h}$ there exist balls $B\left(x_{0}, \sigma_{0} h\right)$ and $B\left(x_{1}, \sigma_{1} h\right)$ with $B\left(x_{0}, \sigma_{0} h\right) \subset T \subset B\left(x_{1}, \sigma_{1} h\right)$ (see [C] for details). Moreover we assume that all nodes in $\partial \Omega_{h}$ are contained in $\partial \Omega$. If $\Omega$ is a domain of class $C^{1, \sigma}$, then $\operatorname{dist}\left(x_{0}, \partial \Omega\right) \leq c h^{1+\sigma}$ for all $x_{0} \in \partial \Omega_{h}$, where $c$ is independent of $h$. For a given triangulation $\mathcal{T}_{h}$ we define $S^{h}\left(\Omega_{h}\right)$ as the space of all globally continuous functions which are affine on the simplices $T \in \mathcal{T}_{h}$, and we denote by $S_{0}^{h}$ the subspace of all functions in $S^{h}$ whose trace on $\partial \Omega_{h}$ is zero. We use the standard notation for the Lebesgue spaces $L^{p}$, the Sobolev spaces $W^{k, p}$ and the Hölder spaces $C^{k, \sigma}$ with norms $\|\cdot\|_{p ; \Omega},\|\cdot\|_{k, p ; \Omega}$ and $\|\cdot\|_{k, \sigma ; \Omega}$, respectively. See Section 3 for the definition of the Morrey and Campanato spaces and their fundamental properties.

In our proofs, we will use two interpolation operators onto $S^{h}$ : the standard interpolation operator $\Pi_{1}$, defined as the linear interpolation of the nodal values of a (continuous) function, and the operator $\Pi_{S Z}$ constructed in [SZ], which is based on local averages. If $W^{2, p}(\Omega) \hookrightarrow C^{0}(\Omega)$, then

$$
\left\|w-\Pi_{1} w\right\|_{\ell, p ; T} \leq c h^{2-\ell}\left\|D^{2} w\right\|_{p ; T}
$$

for all $w \in W^{2, p}\left(\Omega_{h}\right)$, while

$$
\left\|w-\Pi_{S Z} w\right\|_{\ell, 2 ; T} \leq c h^{2-\ell}\left\|D^{2} w\right\|_{2 ; S(T)},
$$

where $S(T)=\bigcup\left\{T^{\prime}: \bar{T}^{\prime} \cap \bar{T} \neq \emptyset\right\}$ for all $w \in W^{2,2}\left(\Omega_{h}\right)$.

In this paper we study general elliptic systems of second order of the form

$$
-D_{\alpha}\left(A_{i j}^{\alpha \beta} D_{\beta} u^{j}\right)=-D_{\alpha} F_{i}^{\alpha}+f_{i}, \quad i=1, \ldots, m,
$$

where the coefficients $A_{i j}^{\alpha \beta}$ satisfy the Legendre-Hadamard condition

$$
A_{i j}^{\alpha \beta} \xi_{\alpha} \eta^{i} \xi_{\beta} \eta^{j} \geq c|\xi|^{2}|\eta|^{2} \quad \forall \xi \in \mathbb{R}^{n}, \eta \in \mathbb{R}^{m} .
$$

Here we use the summation convention. However, our analysis does not include general systems which are elliptic in the sense of Agmon, Douglis and Nirenberg or saddle point problems. The corresponding weak formulation is given by

$$
a(u, \psi)=\mathcal{F}(\psi) \quad \forall \psi \in W_{0}^{1,2}(\Omega)
$$


with

$$
\mathcal{F}(\psi)=\int_{\Omega}\left(F_{i}^{\alpha} D_{\alpha} \psi^{i}+f_{i} \psi^{i}\right) d x
$$

We say that $u_{h} \in S_{0}^{h}$ is a finite element solution of the system if

$$
a\left(u_{h}, \psi_{h}\right)=\mathcal{F}\left(\psi_{h}\right) \quad \forall \psi \in S_{0}^{h}\left(\Omega_{h}\right) .
$$

Here the bilinear form $a(\cdot, \cdot)$ on $W^{1,2} \times W^{1,2}$ associated with $A$ is given by

$$
a(u, v)=\int_{\Omega} A_{i j}^{\alpha \beta} D_{\beta} u^{j} D_{\alpha} v^{i} d x
$$

and we will use $a_{h}$ to denote the bilinear form $a$ restricted to $\Omega_{h}$ :

$$
a_{h}(u, v)=\int_{\Omega_{h}} A_{i j}^{\alpha \beta} D_{\beta} u^{j} D_{\alpha} v^{i} d x .
$$

The following result is a standard result in elliptic theory and can be found for example in [Gi], Teorema 10.1.

Theorem 2.1 (Gårding's inequality). Assume that the coefficients $A_{i j}^{\alpha \beta}$ are uniformly continuous in $\bar{\Omega}$ and satisfy (2.4).

i) If the coefficients are constant, then there exists a constant $\mu>0$ such that

$$
\int_{\Omega} A_{i j}^{\alpha \beta} D_{\beta} \varphi^{j} D_{\alpha} \varphi^{i} d x \geq \mu \int_{\Omega}|D \varphi|^{2} d x \quad \forall \varphi \in W_{0}^{1,2}(\Omega) .
$$

ii) There exists an $R_{0}>0$ such that (2.7) holds for all $\varphi$ with $\operatorname{diam}(\operatorname{spt} \varphi)<R_{0}$.

iii) There exist constants $\nu, H>0$ such that

$$
\int_{\Omega} A_{i j}^{\alpha \beta} D_{\beta} \varphi^{j} D_{\alpha} \varphi^{i} d x \geq \nu \int_{\Omega}|D \varphi|^{2} d x-H \int_{\Omega}|\varphi|^{2} d x \quad \forall \varphi \in W_{0}^{1,2}(\Omega) .
$$

Throughout the paper all constants in the estimates depend only an $n, m, \Omega, A$ and the constant in Gårding's inequality (here we adopt the point of view that the constants in the other usual inequalities like Poincaré's inequality or the Sobolev embedding theorem depend only on these quantities). In particular, they are independent of $h, u, F, f$ and the center $x_{0}$ of the balls $\Omega\left(x_{0}, R\right)$.

\section{Elliptic Regularity in Campanato spaces}

Assume that $\Omega \subset \mathbb{R}^{n}$ is an open domain, $1 \leq p<\infty$ and $\lambda \geq 0$. We define the Morrey space $L^{p, \lambda}(\Omega)$ as the space of all functions $u: \Omega \rightarrow \mathbb{R}^{m}$ such that $u \in L^{p}(\Omega)$ and

$$
\|u\|_{L^{p, \lambda}(\Omega)}^{p}=\sup _{x_{0} \in \bar{\Omega}} \sup _{0<\varrho<\operatorname{diam}(\Omega)} \frac{1}{\varrho^{\lambda}} \int_{\Omega\left(x_{0}, \varrho\right)}|u|^{p} d x<\infty .
$$

The Campanato space $\mathcal{L}^{p, \lambda}(\Omega)$ is the space of all functions $u \in L^{p}(\Omega)$ for which

$$
[u]_{p, \lambda}^{p}=\sup _{x_{0} \in \bar{\Omega}} \sup _{0<\varrho<\operatorname{diam}(\Omega)} \frac{1}{\varrho^{\lambda}} \int_{\Omega\left(x_{0}, \varrho\right)}\left|u-(u)_{x_{0}, \varrho}\right|^{p} d x<\infty .
$$

Here $(u)_{x_{0}, R}=(u)_{\Omega\left(x_{0}, R\right)}$ denotes the mean value of $u$ on $\Omega\left(x_{0}, R\right)$ :

$$
(u)_{x_{0}, R}=\frac{1}{\left|\Omega\left(x_{0}, R\right)\right|} \int_{\Omega\left(x_{0}, R\right)} u d x .
$$


We endow $\mathcal{L}^{p, \lambda}(\Omega)$ with the norm $\|u\|_{\mathcal{L}^{p, \lambda}(\Omega)}=\|u\|_{p ; \Omega}+[u]_{p, \lambda}$. Defined in such a way, the Morrey and Campanato spaces are Banach spaces, and $L^{p, \lambda}(\Omega)$ is isomorphic to $\mathcal{L}^{p, \lambda}(\Omega)$ if $0 \leq \lambda<n$ and the domain $\Omega$ is sufficiently smooth (in general one needs that $\Omega$ is a domain of type $A$; see [Ca1] for the precise definition). Moreover, $\mathcal{L}^{p, \lambda}(\Omega)$ is isomorphic to $C^{0, \sigma}(\bar{\Omega})$ with $\sigma=\frac{\lambda-n}{p}$ for $\lambda \in(n, n+p]$, while $\mathcal{L}^{p, n}(\Omega)$ is isomorphic to $\operatorname{BMO}(\Omega)$, the space of functions with bounded mean oscillation which was defined in the fundamental paper by John and Nirenberg [JN]. For more information about these spaces, see e.g. [KJF].

Starting from Campanato's paper [Ca1], a complete regularity theory for elliptic equations and systems has been developed (see, e.g. [Ca2], [Gi], [G]). We summarize the relevant results in the following two theorems (see [Gi], Capitolo 10). Part iii) in Theorem 3.1 follows by contradiction from the estimates in parts $i$ ) and $i i$ ), since in this situation the homogeneous equation has only the trivial solution. Throughout the paper we write $\lambda-2$ instead of $(\lambda-2)^{+}=\max \{\lambda-2,0\}$.

Theorem 3.1. Assume that the coefficients $A_{i j}^{\alpha \beta}$ satisfy the Legendre-Hadamard condition (2.4). Let $u \in W_{0}^{1,2}(\Omega)$ be a weak solution of (2.5).

i) Suppose $\Omega$ is a domain of class $C^{1}$ and that $A_{i j}^{\alpha \beta} \in C^{0}(\bar{\Omega})$. If $\lambda \in[0, n)$, $F \in L^{2, \lambda}(\Omega)$ and $f \in L^{2, \lambda-2}(\Omega)$, then $D u \in L^{2, \lambda}(\Omega)$ and we have the a priori estimate

$$
\|D u\|_{L^{2, \lambda}(\Omega)} \leq c\left(\|u\|_{2 ; \Omega}+\|f\|_{L^{2, \lambda-2}(\Omega)}+\|F\|_{L^{2, \lambda}(\Omega)}\right) .
$$

ii) Suppose that $\Omega$ is a domain of class $C^{1, \sigma}$ and $A_{i j}^{\alpha \beta} \in C^{0, \sigma}(\bar{\Omega})$. If $F \in \mathcal{L}^{2, \lambda}(\Omega)$ and $f \in L^{2, \lambda-2}(\Omega)$ with $\lambda \leq n+2 \sigma$, then $D u \in \mathcal{L}^{2, \lambda}(\Omega)$ and we have

$$
\|D u\|_{\mathcal{L}^{2, \lambda}(\Omega)} \leq c\left(\|u\|_{2 ; \Omega}+\|f\|_{L^{2, \lambda-2}(\Omega)}+\|F\|_{\mathcal{L}^{2, \lambda}(\Omega)}\right) .
$$

iii) If the system has a unique solution, then the a priori estimates in i) and ii) hold without the norm of $u$ on the right hand side.

A similar result holds for the higher derivatives of $u$.

Theorem 3.2. Assume $\Omega$ is a domain of class $C^{k+1}\left(C^{k+1, \sigma}\right)$ and that the coeffcients $A_{i j}^{\alpha \beta} \in C^{k}(\bar{\Omega})\left(C^{k, \sigma}(\bar{\Omega})\right)$ satisfy the Legendre-Hadamard condition (2.4). Let $u \in W_{0}^{1,2}(\Omega)$ be a weak solution of $(2.5)$ and $k \geq 1$. Suppose that $D^{k} F \in L^{2, \lambda}(\Omega)$ (resp. $\mathcal{L}^{2, \lambda}(\Omega)$ ) and $D^{k-1} f \in L^{2, \lambda}(\Omega)$ with $\lambda \in(0, n)$ (resp. $\mathcal{L}^{2, \lambda}(\Omega)$ with $\lambda \leq$ $n+2 \sigma)$. Then $D^{k+1} u \in L^{2, \lambda}(\Omega)$ (resp. $\mathcal{L}^{2, \lambda}(\Omega)$ ), and we have the corresponding a priori estimates.

The main ingredient in the proof of these regularity results is local decay estimates for the solution of the homogeneous system, which we will refer to as Campanato inequalities.

Proposition 3.3. Assume that the coefficients $A_{i j}^{\alpha \beta}\left(x_{0}\right)$ satisfy (2.4) and that $v$ is a solution of the homogeneous system $D_{\alpha}\left(A_{i j}^{\alpha \beta}\left(x_{0}\right) D_{\beta} v^{j}\right)=0$ in $\Omega\left(x_{0}, R\right)$.

i) If $\Omega\left(x_{0}, R\right) \subset \Omega$, then for all $0<\varrho<R$

$$
\int_{\Omega\left(x_{0}, \varrho\right)}|D v|^{2} d x \leq c\left(\frac{\varrho}{R}\right)^{n} \int_{\Omega\left(x_{0}, R\right)}|D v|^{2} d x,
$$


and

$$
\int_{\Omega\left(x_{0}, \varrho\right)}\left|D v-(D v)_{x_{0}, \varrho}\right|^{2} d x \leq c\left(\frac{\varrho}{R}\right)^{n+2} \int_{\Omega\left(x_{0}, R\right)}\left|D v-(D v)_{x_{0}, R}\right|^{2} d x .
$$

ii) If $x_{0} \in \partial \Omega$ and $\Omega\left(x_{0}, R\right)=B\left(x_{0}, R\right)^{+}$with $v=0$ on $\Gamma_{R}$, then

$$
\int_{\Omega\left(x_{0}, \varrho\right)}|D v|^{2} d x \leq c\left(\frac{\varrho}{R}\right)^{n} \int_{\Omega\left(x_{0}, R\right)}|D v|^{2} d x
$$

and

$$
\int_{\Omega\left(x_{0}, \varrho\right)}\left|D v-\left(D_{n} v\right)_{x_{0}, \varrho} \otimes e_{n}\right|^{2} d x \leq c\left(\frac{\varrho}{R}\right)^{n+2} \int_{\Omega\left(x_{0}, R\right)}\left|D v-\left(D_{n} v\right)_{x_{0}, R} \otimes e_{n}\right|^{2} d x .
$$

Remark. For systems with continuous coefficients one obtains a similar estimate with an additional term $\omega^{2}(R) \int_{\Omega\left(x_{0}, R\right)}|D v|^{2} d x$ on the right hand side, where $\omega$ denotes the oscillation of the coefficients on $\Omega\left(x_{0}, R\right)$.

We define the following discrete analogues of the Morrey spaces $L^{2, \lambda}(\Omega)$ and the Campanato spaces $\mathcal{L}^{2, \lambda}(\Omega)$, where the radii in the definition are bounded from below by $h$. A function $u$ belongs to the discrete Morrey space $L_{h}^{p, \lambda}(\Omega)$ if

$$
\|u\|_{L_{h}^{p, \lambda}}^{p}=\sup _{x_{0} \in \bar{\Omega} h<\varrho<\operatorname{diam}(\Omega)} \sup _{\varrho^{\lambda}} \int_{\Omega\left(x_{0}, \varrho\right)}|u|^{p} d x<\infty,
$$

and to the discrete Campanato space $\mathcal{L}_{h}^{p, \lambda}(\Omega)$ if

$$
[u]_{p, \lambda ; h}^{p}=\sup _{x_{0} \in \bar{\Omega}} \sup _{h<\varrho<\operatorname{diam}(\Omega)} \frac{1}{\varrho^{\lambda}} \int_{\Omega\left(x_{0}, \varrho\right)}\left|u-(u)_{x_{0}, \varrho}\right|^{p} d x<\infty .
$$

The following lemma shows that a function $u_{h} \in S^{h}$ is bounded in $L^{2, \lambda}\left(\Omega_{h}\right)$ if and only if it is bounded in $L_{h}^{2, \lambda}\left(\Omega_{h}\right)$.

Lemma 3.4. Let $u_{h} \in S^{h}, h>0$ small enough, and $0 \leq \lambda \leq n$.

i) If $u_{h} \in L_{h}^{2, \lambda}\left(\Omega_{h}\right)$ with $\|u\|_{L_{h}^{2, \lambda}} \leq C$, then $u_{h} \in L^{2, \lambda}\left(\Omega_{h}\right)$ and $\left\|u_{h}\right\|_{L^{2, \lambda}} \leq \tilde{C}$, where $\tilde{C}$ depends only $\sigma_{0}, \lambda, n$ and $C$.

ii) The same statement holds also for $\mathcal{L}_{h}^{2, \lambda}\left(\Omega_{h}\right)$.

Proof. Assume that $0<\varrho \leq h$ and let $T_{i}, i=1, \ldots, L$, be the triangles $T \in \mathcal{T}_{h}$ such that $T \cap \Omega_{h}\left(x_{0}, \varrho\right) \neq \emptyset$. To prove $\left.i\right)$, choose points $x_{i} \in T_{i}$ such that $\Omega_{h}\left(x_{i}, \sigma_{0} h\right) \subset$ $T_{i}$. Then

$$
\begin{aligned}
& \frac{1}{\varrho^{\lambda}} \int_{\Omega_{h}\left(x_{0}, \varrho\right)}\left|D u_{h}\right|^{2} d x \\
& \quad \leq \sum_{i=1}^{L} \frac{\left|T_{i} \cap \Omega_{h}\left(x_{0}, \varrho\right)\right|^{\lambda / n}}{\varrho^{\lambda}} \frac{\left|T_{i} \cap \Omega_{h}\left(x_{i}, \varrho\right)\right|^{1-\lambda / n}}{\left(\sigma_{0} h\right)^{n-\lambda}} \frac{1}{\left(\sigma_{0} h\right)^{\lambda}} \int_{\Omega_{h}\left(x_{i}, \sigma_{0} h\right)}\left|D u_{h}\right|^{2} d x \\
& \quad \leq c L C,
\end{aligned}
$$

where $c$ depends only on $\sigma_{0}, \lambda$ and $n$. To prove $\left.i i\right)$, choose for a given domain $\Omega_{h}\left(x_{0}, \varrho\right)$ the smallest radius $\tilde{\varrho}$ such that $\Omega_{h}\left(x_{0}, \varrho \varrho\right)$ contains all triangles $T_{i} \in \mathcal{T}_{h}$ 
defined above. If $x_{0} \in \Omega_{h}$ and $\Omega_{h}\left(x_{0}, \tilde{\varrho}\right) \cap \partial \Omega_{h} \neq \emptyset$, then replace $\Omega_{h}\left(x_{0}, \tilde{\varrho}\right)$ by a domain $\Omega_{h}\left(\bar{x}_{0}, \bar{\varrho}\right)$ such that $\bar{\varrho} \leq \operatorname{ch}$ and $\Omega_{h}\left(x_{0}, \tilde{\varrho}\right) \subset \Omega_{h}\left(\bar{x}_{0}, \bar{\varrho}\right)$. Otherwise define $\bar{x}_{0}=x_{0}$ and $\bar{\varrho}=\tilde{\varrho}$. Then we conclude, as in case $i$ ), that

$$
\begin{aligned}
\frac{1}{\varrho^{\lambda}} \int_{\Omega_{h}\left(x_{0}, \varrho\right)}\left|D u_{h}-\left(D u_{h}\right)_{x_{0}, \varrho}\right|^{2} d x & \leq \frac{c}{\varrho^{\lambda}} \int_{\Omega_{h}\left(x_{0}, \varrho\right)}\left|D u_{h}-\xi\right|^{2} d x \\
& \leq \frac{c}{\bar{\varrho}^{\lambda}} \int_{\Omega_{h}\left(\bar{x}_{0}, \bar{\varrho}\right)}\left|D u_{h}-\xi\right|^{2} d x,
\end{aligned}
$$

and the assertion follows with $\xi=\left(D u_{h}\right)_{\bar{x}_{0}, \bar{\varrho}}$.

\section{A pointwise interior estimate}

The main result in this section is the pointwise estimate in Proposition 4.8. It is based on the following analogues of the Campanato inequalities in Section 3 for the finite element solution $u_{h}$ on balls $\Omega\left(x_{0}, R\right) \subset \Omega$. Throughout the rest of the paper we will set

$$
\mathcal{R}_{h}(F, f ; R)=\int_{\Omega_{h}\left(x_{0}, R\right)}\left|F-(F)_{\Omega_{h}\left(x_{0}, R\right)}\right|^{2} d x+R^{2} \int_{\Omega_{h}\left(x_{0}, R\right)}|f|^{2} d x,
$$

and $\eta$ denotes a nonnegative, continuous function such that $\eta(t) \leq c t^{1 / n}$ for $n \geq 3$ and $\eta(t) \leq c(\mu) t^{\mu}$ for all $\mu \in\left(0, \frac{1}{2}\right)$ for $n=2$. We denote the modulus of continuity of the coefficients by $\omega$, i.e.

$$
\omega(R)=\sup _{\left|x-x_{0}\right|<R} \sup _{\alpha, \beta=1, \ldots, n} \sup _{i, j=1, \ldots, m}\left|A_{i j}^{\alpha \beta}(x)-A_{i j}^{\alpha \beta}\left(x_{0}\right)\right| .
$$

Lemma 4.1. There exists a constant $\Lambda>0$ such that for all $h \leq \varrho \leq R \leq R_{0}$ and $R \geq \Lambda h$ the following inequalities hold:

$$
\begin{gathered}
\int_{\Omega\left(x_{0}, \varrho\right)}\left|D u_{h}\right|^{2} d x \leq c\left\{\left(\frac{\varrho}{R}\right)^{n}+\omega^{2}(R)+\eta\left(\frac{h}{R}\right)\right\} \int_{\Omega\left(x_{0}, R\right)}\left|D u_{h}\right|^{2} d x+c \mathcal{R}_{h}(F, f ; R), \\
\int_{\Omega\left(x_{0}, \varrho\right)}\left|D u_{h}-\left(D u_{h}\right)_{x_{0}, \varrho}\right|^{2} d x \leq c\left\{\left(\frac{\varrho}{R}\right)^{n+2}+\eta\left(\frac{h}{R}\right)\right\} \int_{\Omega\left(x_{0}, R\right)}\left|D u_{h}-\left(D u_{h}\right)_{x_{0}, R}\right|^{2} d x \\
+c \omega^{2}(R) \int_{\Omega\left(x_{0}, R\right)}\left|D u_{h}\right|^{2} d x+c \mathcal{R}_{h}(F, f ; R) .
\end{gathered}
$$

Here $\Lambda$ is independent of $x_{0}, h, \varrho, R, u$ and $u_{h}$.

We split the proof into a series of lemmas. The idea is to decompose $u_{h}$ as a sum $\left(u_{h}-w\right)+w$, where $w \in W^{1,2}\left(\Omega\left(x_{0}, R\right)\right)$ is the solution of the homogeneous system with constant coefficients

$$
\begin{array}{rlll}
a_{0}(w, \psi) & =0 & \forall \psi \in W_{0}^{1,2}\left(\Omega\left(x_{0}, R\right)\right), \\
w=u_{h} & \text { on } \partial \Omega\left(x_{0}, R\right),
\end{array}
$$


and to use the Campanato estimate in Proposition 3.3 for $w$. Here $a_{0}$ denotes the bilinear form with constant coefficients $A_{i j}^{\alpha \beta}\left(x_{0}\right)$. It follows from $w=u_{h}$ on $\partial \Omega\left(x_{0}, R\right)$ and the divergence theorem that

$$
\int_{\Omega\left(x_{0}, R\right)} D_{\alpha} w^{i} d x=\int_{\Omega\left(x_{0}, R\right)} D_{\alpha} u_{h}^{i} d x, \quad i=1, \ldots, m, \alpha=1, \ldots, n .
$$

We summarize the important properties of $w$ in the following lemma.

Lemma 4.2. Assume that $w$ is the solution of (4.2).

i) We have the a priori estimate

$$
\int_{\Omega\left(x_{0}, R\right)}|D w-\xi|^{2} d x \leq c \int_{\Omega\left(x_{0}, R\right)}\left|D u_{h}-\xi\right|^{2} d x \quad \forall \xi \in \mathbb{R}^{m n} .
$$

ii) We have for $k \geq 2$ the Caccioppoli estimate

$$
\int_{\Omega\left(x_{0}, \varrho\right)}\left|D^{k} w\right|^{2} d x \leq \frac{c}{(R-\varrho)^{2(k-1)}} \int_{\Omega\left(x_{0}, R\right)}|D w-\xi|^{2} d x \quad \forall \xi \in \mathbb{R}^{m n} .
$$

iii) We have the pointwise estimate

$$
\sup _{x \in \Omega\left(x_{0}, \varrho\right)}\left|D^{k} w\right|^{2} \leq \frac{c}{(R-\varrho)^{2(k-1)}} \frac{1}{R^{n}} \int_{\Omega\left(x_{0}, R\right)}|D w-\xi|^{2} d x \quad \forall \xi \in \mathbb{R}^{m n} .
$$

Proof. In view of Gårding's inequality we obtain $i)$ from $a_{0}\left(u_{h}-w, u_{h}-w\right)=$ $a_{0}\left(u_{h}, u_{h}-w\right)$. The Caccioppoli estimate in $\left.i i\right)$ is standard (see, e.g. [Gi]), and the pointwise estimate follows from ii) by Sobolev's embedding theorem.

In order to obtain an estimate for $D u_{h}-D w$ we define

$$
\psi=\zeta^{2}\left(u_{h}-w\right), \quad \psi_{h}=\Pi_{1} \psi,
$$

where $\zeta \geq 0$ is a smooth cut-off function such that $\zeta=\Pi_{1} \zeta=0$ on $\mathbb{R}^{n} \backslash \Omega\left(x_{0}, \frac{3}{4} R\right)$, $\zeta=1$ on $\Omega\left(x_{0}, \frac{R}{2}\right)$, and $\left\|D^{i} \zeta\right\|_{\infty} \leq c R^{-i}$ for $i=1,2$ ( $R$ will be of order one, and the existence of $\zeta$ is thus clear for $h$ small enough). The following estimate of the difference $\psi-\psi_{h}$ in the energy norm will be important. For $n \leq 3$ an estimate of this type follows easily from the interpolation estimate (2.1). In arbitrary dimensions, however, a direct computation is necessary.

Lemma 4.3. Let $\psi$ and $\psi_{h}$ be defined as in (4.4).

i) We have the local estimate

$$
\int_{T}\left|D \psi-D \psi_{h}\right|^{2} d x \leq \sup _{x \in T} \zeta^{2}(x)\left\{\frac{c}{R^{2}} \int_{T}\left|u_{h}-w\right|^{2} d x+c h^{2}|T| \sup _{x \in T}\left|D^{2} w(x)\right|^{2}\right\} .
$$

ii) We have for all $\xi \in \mathbb{R}^{m n}$ the global estimate

$$
\int_{\Omega\left(x_{0}, R\right)}\left|D \psi-D \psi_{h}\right|^{2} d x \leq \frac{c}{R^{2}} \int_{\Omega\left(x_{0}, R\right)}\left|u_{h}-w\right|^{2} d x+c \frac{h^{2}}{R^{2}} \int_{\Omega\left(x_{0}, R\right)}|D w-\xi|^{2} d x
$$


Proof. Clearly $i i$ ) follows from $i$ ) by Lemma 4.2 . To prove $i$ ), let $a_{i}, i=1, \ldots, n+$ 1 , be the nodes of the simplex $T$ and $\Phi_{i}$ the standard nodal basis of $T$, i.e., $\Phi_{i}\left(a_{j}\right)=\delta_{i j}$. Then $\Pi_{1} w(x)=\sum_{i=1}^{n+1} \Phi_{i}(x) w\left(a_{i}\right)$ for all $w \in C^{0}(T)$. We have $D \psi=2 \zeta D \zeta\left(u_{h}-w\right)+\zeta^{2}\left(D u_{h}-D w\right)$, and thus

$$
\begin{aligned}
\int_{T}\left|D \psi-D \psi_{h}\right|^{2} \leq & \frac{c}{R^{2}} \sup _{x \in T} \zeta^{2}(x) \int_{T}\left|u_{h}-w\right|^{2} d x+c \int_{T} \zeta^{2}\left|D w-D \Pi_{1} w\right|^{2} d x \\
& +c \int_{T}\left|\zeta^{2}\left(D u_{h}-D \Pi_{1} w\right)-D \psi_{h}\right|^{2} d x .
\end{aligned}
$$

The second term is estimated by the interpolation inequality $(2.1)$ with $(p=\infty)$, while by definition of $\psi_{h}$

$$
\begin{aligned}
\int_{T} \mid \zeta^{2}\left(D u_{h}\right. & \left.-D \Pi_{1} w\right)-\left.D \psi_{h}\right|^{2} d x \\
& =\int_{T}\left|\sum_{i=1}^{n+1} D \Phi_{i}(x)\left(u_{h}-w\right)\left(a_{i}\right)\left(\zeta^{2}(x)-\zeta^{2}\left(a_{i}\right)\right)\right|^{2} d x \\
& \leq \sum_{i=1}^{n+1} \sup _{x \in T}\left|D \Phi_{i}(x)\right|^{2}\left|\left(u_{h}-w\right)\left(a_{i}\right)\right|^{2}|T|\left(\sup _{x \in T}\left|\zeta^{2}(x)-\zeta^{2}\left(a_{i}\right)\right|\right)^{2} .
\end{aligned}
$$

By assumption $\sup _{x \in \Omega_{h}}\left|D \Phi_{i}(x)\right| \leq c h^{-1}$ and $\sup _{x \in T}\left|\zeta(x)-\zeta\left(a_{i}\right)\right| \leq c\left|x-a_{i}\right| / R$; therefore

$$
\sup _{x \in T}\left|\zeta^{2}(x)-\zeta^{2}\left(a_{i}\right)\right| \leq c \frac{\left|x-a_{i}\right|}{R} \sup _{x \in T}\left|\zeta(x)+\zeta\left(a_{i}\right)\right| \leq 2 c \frac{\operatorname{diam}(T)}{R} \sup _{x \in T}|\zeta| .
$$

Since $\int_{T}\left|v_{h}\right|^{2} d x$ and $|T| \sum_{i=1}^{n+1}\left|v_{h}\left(a_{i}\right)\right|^{2}$ are equivalent norms on $T$, we obtain

$$
\int_{T}\left|\zeta^{2}\left(D u_{h}-D \Pi_{1} w\right)-D \psi_{h}\right|^{2} d x \leq \frac{c}{R^{2}} \sup _{x \in T} \zeta^{2} \int_{T}\left|u_{h}-\Pi_{1} w\right|^{2} d x
$$

and the assertion of the lemma follows easily.

By (2.6) and (4.2) we obtain, since $\psi_{h} \in S_{0}^{h}$,

$$
a\left(u_{h}-w, \psi\right)=a\left(u_{h}-w, \psi-\psi_{h}\right)+\mathcal{F}\left(\psi_{h}\right)-\left(a-a_{0}\right)\left(w, \psi_{h}\right) .
$$

We estimate the different terms in the following lemmas.

Lemma 4.4. We have

$$
a\left(u_{h}-w, \psi\right) \geq c \int_{\Omega\left(x_{0}, R\right)} \zeta^{2}\left|D u_{h}-D w\right|^{2} d x-\frac{c}{R^{2}} \int_{\Omega\left(x_{0}, R\right)}\left|u_{h}-w\right|^{2} d x,
$$

where the constant $c>0$ depends on the constant in Gärding's inequality.

Proof. A direct computation shows that

$$
\begin{aligned}
& A_{i j}^{\alpha \beta} D_{\beta}\left(u_{h}^{j}-w^{j}\right) D_{\alpha}\left(\zeta^{2}\left(u_{h}^{i}-w^{i}\right)\right)=A_{i j}^{\alpha \beta} D_{\beta}\left(\zeta\left(u_{h}^{j}-w^{j}\right)\right) D_{\alpha}\left(\zeta\left(u_{h}^{i}-w^{i}\right)\right) \\
& \quad+A_{i j}^{\alpha \beta} D_{\beta}\left(u_{h}^{j}-w^{j}\right) D_{\alpha} \zeta\left(\zeta\left(u_{h}^{i}-w^{i}\right)\right)-A_{i j}^{\alpha \beta} D_{\beta} \zeta\left(u_{h}^{j}-w^{j}\right) D_{\alpha}\left(\zeta\left(u_{h}^{i}-w^{i}\right)\right) .
\end{aligned}
$$

By Gårding's inequality

$$
\begin{aligned}
a\left(u_{h}-w, \psi\right) \geq c & \int_{\Omega}\left|D\left(\zeta\left(u_{h}-w\right)\right)\right|^{2} d x-(m n)|A|_{\infty}|D \zeta|_{\infty}\left\|u_{h}-w\right\|_{2 ; \Omega\left(x_{0}, R\right)} \\
& \cdot\left\{\left\|\zeta\left(D u_{h}-D w\right)\right\|_{2 ; \Omega\left(x_{0}, R\right)}+\left\|D\left(\zeta\left(u_{h}-w\right)\right)\right\|_{2 ; \Omega\left(x_{0}, R\right)}\right\},
\end{aligned}
$$

and the assertion of the lemma follows easily from Young's inequality. 
Lemma 4.5. We have for $\varepsilon>0$

$$
\begin{aligned}
\left|a\left(u_{h}-w, \psi-\psi_{h}\right)\right| \leq & \varepsilon \int_{\Omega\left(x_{0}, R\right)} \zeta^{2}\left|D u_{h}-D w\right|^{2} d x \\
& +c\left\{\frac{h}{R} \int_{\Omega\left(x_{0}, R\right)}\left|D u_{h}-\xi\right|^{2} d x+\frac{1}{\varepsilon R^{2}} \int_{\Omega\left(x_{0}, R\right)}\left|u_{h}-w\right|^{2} d x\right\} .
\end{aligned}
$$

Proof. Choose for each triangle $T \in \mathcal{T}_{h}$ a point $x_{T}$ such that $\sup _{x \in T} \zeta(x)=\zeta\left(x_{T}\right)$. We have, by Hölder's inequality and Lemma 4.3,

$$
\begin{aligned}
\mid a\left(u_{h}\right. & \left.-w, \psi-\psi_{h}\right) \mid \\
& \leq(m n)|A|_{\infty} \sum_{T \cap \operatorname{spt} \zeta \neq \emptyset}\left\|D u_{h}-D w\right\|_{2 ; T}\left\|D \psi-D \psi_{h}\right\|_{2 ; T} \\
& \leq c \sum_{T \cap \operatorname{spt} \zeta \neq \emptyset}\left\|D u_{h}-D w\right\|_{2 ; T}\left\{\frac{\zeta\left(x_{T}\right)}{R}\left\|u_{h}-w\right\|_{2 ; T}+h|T|^{1 / 2} \sup _{x \in T}\left|D^{2} w(x)\right|\right\} .
\end{aligned}
$$

By definition

$$
\begin{aligned}
\zeta\left(x_{T}\right)\left\|D u_{h}-D w\right\|_{2 ; T} \leq\left\|\left(\zeta\left(x_{T}\right)-\zeta\right)\left(D u_{h}-D w\right)\right\|_{2 ; T}+\left\|\zeta\left(D u_{h}-D w\right)\right\|_{2 ; T} \\
\quad \leq \sup _{x \in T} \frac{\left|\zeta(x)-\zeta\left(x_{T}\right)\right|}{\left|x-x_{T}\right|}\left|x-x_{T}\right|\left\|D u_{h}-D w\right\|_{2 ; T}+\left\|\zeta\left(D u_{h}-D w\right)\right\|_{2 ; T} \\
\leq c \frac{\operatorname{diam}(T)}{R}\left\|D u_{h}-D w\right\|_{2 ; T}+\left\|\zeta\left(D u_{h}-D w\right)\right\|_{2 ; T},
\end{aligned}
$$

and therefore we obtain

$$
\begin{aligned}
& \sum_{T \cap \operatorname{spt} \zeta \neq \emptyset}\left\|D u_{h}-D w\right\|_{2 ; T} \frac{\zeta\left(x_{T}\right)}{R}\left\|u_{h}-w\right\|_{2 ; T} \\
\leq & c \sum_{T \cap \operatorname{spt} \zeta \neq \emptyset} \frac{h}{R^{2}}\left\|D u_{h}-D w\right\|_{2 ; T}\left\|u_{h}-w\right\|_{2 ; T}+\frac{1}{R}\left\|\zeta\left(D u_{h}-D w\right)\right\|_{2 ; T}\left\|u_{h}-w\right\|_{2 ; T} \\
\leq & \varepsilon \int_{\Omega\left(x_{0}, R\right)} \zeta^{2}\left|D u_{h}-D w\right|^{2} d x+c \frac{h^{2}}{R^{2}} \int_{\Omega\left(x_{0}, R\right)}\left|D u_{h}-D w\right|^{2} d x+\frac{c}{\varepsilon R^{2}} \int_{\Omega\left(x_{0}, R\right)}\left|u_{h}-w\right|^{2} d x .
\end{aligned}
$$

On the other hand, we obtain by Lemma 4.2

$$
\begin{aligned}
\sum_{T \cap \text { spt } \zeta \neq \emptyset} & \left\|D u_{h}-D w\right\|_{2 ; T} h|T|^{1 / 2} \sup _{x \in T}\left|D^{2} w\right| \\
\leq & c \frac{h}{R} \int_{\Omega\left(x_{0}, R\right)}\left|D u_{h}-D w\right|^{2} d x+c h R\left|\Omega\left(x_{0}, R\right)\right| \sup _{x \in \Omega\left(x_{0}, \frac{3}{4} R\right)}\left|D^{2} w\right|^{2} \\
\leq & c \frac{h}{R} \int_{\Omega\left(x_{0}, R\right)}\left|D u_{h}-D w\right|^{2} d x+c \frac{h}{R} \int_{\Omega\left(x_{0}, R\right)}|D w-\xi|^{2} d x,
\end{aligned}
$$

and the assertion of the lemma follows easily in view of Lemma 4.2. 
Lemma 4.6. We have

$$
\begin{aligned}
\mid \mathcal{F}\left(\psi_{h}\right)- & \left(a-a_{0}\right)\left(w, \psi_{h}\right) \mid \\
\leq & \frac{c}{\varepsilon}\left\{\omega^{2}(R) \int_{\Omega\left(x_{0}, R\right)}|D w|^{2} d x+\mathcal{R}_{h}(F, f ; R)\right\} \\
& +\varepsilon\left\{\int_{\Omega\left(x_{0}, R\right)} \zeta^{2}\left|D u_{h}-D w\right|^{2} d x\right. \\
& \left.+\frac{1}{R^{2}} \int_{\Omega\left(x_{0}, R\right)}\left|u_{h}-w\right|^{2} d x+\left(\frac{h}{R}\right)^{2} \int_{\Omega\left(x_{0}, R\right)}\left|D u_{h}-\xi\right|^{2} d x\right\} .
\end{aligned}
$$

Proof. Since $\psi_{h} \in W_{0}^{1,2}\left(\Omega_{h}\left(x_{0}, R\right)\right)$, we have by definition

$$
\begin{aligned}
& \left|\mathcal{F}\left(\psi_{h}\right)-\left(a-a_{0}\right)\left(w, \psi_{h}\right)\right| \leq\left\|D \psi_{h}\right\|_{2 ; \Omega\left(x_{0}, R\right)} \\
& \quad \cdot\left(\left\|F-(F)_{\Omega_{h}\left(x_{0}, R\right)}\right\|_{2 ; \Omega_{h}\left(x_{0}, R\right)}+c R\|f\|_{2 ; \Omega_{h}\left(x_{0}, R\right)}+m n \omega(R)\|D w\|_{2 ; \Omega\left(x_{0}, R\right)}\right) .
\end{aligned}
$$

By the triangle inequality

$$
\left\|D \psi_{h}\right\|_{2 ; \Omega}^{2} \leq 2\left\|D \psi-D \psi_{h}\right\|_{2 ; \Omega}^{2}+2\|D \psi\|_{2 ; \Omega}^{2},
$$

and the assertion of the lemma follows easily in view of Lemmas 4.3 and 4.2.

If we combine the inequalities in the above lemmas with the estimates in Lemma 4.2 , we obtain the following inequality:

$$
\begin{aligned}
\int_{\Omega\left(x_{0}, R\right)} \zeta^{2}\left|D u_{h}-D w\right|^{2} d x \\
\leq c\left\{\frac{h}{R} \int_{\Omega\left(x_{0}, R\right)}\left|D u_{h}-\left(D u_{h}\right)_{x_{0}, R}\right|^{2} d x+\mathcal{R}_{h}(F, f ; R)\right. \\
\left.\quad+\frac{1}{R^{2}} \int_{\Omega\left(x_{0}, R\right)}\left|u_{h}-w\right|^{2} d x+\omega^{2}(R) \int_{\Omega\left(x_{0}, R\right)}\left|D u_{h}\right|^{2} d x\right\} .
\end{aligned}
$$

It therefore remains to estimate $\left\|u_{h}-w\right\|$. This is done in the following lemma with a duality argument.

Lemma 4.7. There exists a constant $\Lambda>0$ such that for all $R$ with $R \geq \Lambda h$ the following inequality holds:

$$
\begin{aligned}
\int_{\Omega\left(x_{0}, R\right)}\left|u_{h}-w\right|^{2} d x \leq & \eta\left(\frac{h}{R}\right) R^{2} \int_{\Omega\left(x_{0}, R\right)}\left|D u_{h}-D w\right|^{2} d x \\
& +c R^{2}\left\{\omega^{2}(R) \int_{\Omega\left(x_{0}, R\right)}|D w|^{2} d x+\mathcal{R}_{h}(F, f ; R)\right\} .
\end{aligned}
$$

Remark. Let $v \in W_{0}^{1,2}\left(\Omega\left(x_{0}, R\right)\right)$ be the solution of

$$
-D_{\alpha}\left(A_{i j}^{\alpha \beta} D_{\beta} v^{j}\right)=-D_{\alpha} F_{i}^{\alpha}+f_{i}
$$


with $F \in W^{1,2}\left(\Omega\left(x_{0}, R\right)\right), f \in L^{2}\left(\Omega\left(x_{0}, R\right)\right)$, and Lipschitz continuous coefficients. Then $v \in W^{2,2}\left(\Omega\left(x_{0}, R\right)\right.$ ) (see, e.g., [Gi], Teorema 10.6) and by homogeneity

$$
\begin{aligned}
\|v\|_{2 ; \Omega\left(x_{0}, R\right)}+ & R\|D v\|_{2 ; \Omega\left(x_{0}, R\right)}+R^{2}\left\|D^{2} v\right\|_{2 ; \Omega\left(x_{0}, R\right)} \\
& \leq c\left(R^{2}\|f\|_{2 ; \Omega\left(x_{0}, R\right)}+R\left\|F-(F)_{x_{0}, R}\right\|_{2 ; \Omega\left(x_{0}, R\right)}\right) .
\end{aligned}
$$

This scaling is expressed in the inequality above, since $u_{h}-w \in W_{0}^{1,2}\left(\Omega\left(x_{0}, R\right)\right)$ solves approximately the system

$$
-D_{\alpha}\left(A_{i j}^{\alpha \beta} D_{\beta}\left(u_{h}^{j}-w^{j}\right)\right)=-D_{\alpha}\left(F_{i}^{\alpha}+\left(A_{i j}^{\alpha \beta}-A_{i j}^{\alpha \beta}\left(x_{0}\right)\right) D_{\beta} w^{j}\right)+f_{i} .
$$

Proof. We give the proof for $n \geq 3$ (for $n=2$ use Hölder's inequality to deduce an analogue of (4.7) below). We will show that for $\delta, \varepsilon>0$

$$
\begin{aligned}
& \int_{\Omega\left(x_{0}, R\right)}\left|u_{h}-w\right|^{2} d x \\
& \leq \varepsilon R^{2} \int_{\Omega\left(x_{0}, R\right)}\left|D u_{h}-D w\right|^{2} d x+\left(\frac{c}{\varepsilon}\left(\frac{h}{R}\right)^{2 / n}+\delta\right) \int_{\Omega\left(x_{0}, R\right)}\left|u_{h}-w\right|^{2} d x \\
& \quad+\frac{c}{\delta} R^{2}\left\{\omega^{2}(R) \int_{\Omega\left(x_{0}, R\right)}|D w|^{2} d x+\mathcal{R}_{h}(F, f ; R)\right\} ;
\end{aligned}
$$

the assertion of the lemma follows with $\varepsilon=\left(\frac{h}{R}\right)^{1 / n}$ and $\delta, \frac{h}{R}$ small enough.

Let $z \in W_{0}^{1,2}\left(\Omega\left(x_{0}, R\right)\right)$ be the solution of the adjoint system

$$
-D_{\alpha}\left(A_{j i}^{\beta \alpha} D_{\beta} z^{j}\right)=u_{h}^{i}-w^{i} .
$$

Then $z \in W^{2,2}\left(\Omega\left(x_{0}, R\right)\right)$, and the estimate (4.6) holds with $f=u_{h}-w$ and $F=0$. Let $r \in\left(0, \frac{R}{2}\right)$, and choose a cut-off function $\tau$ such that $\tau=1$ on $\Omega\left(x_{0}, R-r\right), \tau=0$ on $\mathbb{R}^{n} \backslash \Omega\left(x_{0}, R\right)$ and $\left|D^{i} \tau\right|_{\infty} \leq c h^{-i}$ for $i=1,2$. Let $A_{r}=\Omega\left(x_{0}, R\right) \backslash \Omega\left(x_{0}, R-r\right)$. We fix $r=c h$ such that $\tau=\Pi_{S Z} \tau=0$ on $\mathbb{R}^{n} \backslash \Omega\left(x_{0}, R\right)$. By Hölder's inequality, the Sobolev embedding theorem, and (4.6)

$$
\begin{aligned}
\|D z\|_{2 ; A_{r}} & \leq\left|A_{r}\right|^{1 / n}\|D z\|_{2 n /(n-2) ; \Omega\left(x_{0}, R\right)} \\
& \leq c\left|A_{r}\right|^{1 / n}\left(\frac{1}{R}\|D z\|_{2 ; \Omega\left(x_{0}, R\right)}+\left\|D^{2} z\right\|_{2 ; \Omega\left(x_{0}, R\right)}\right) \\
& \leq c R\left(\frac{h}{R}\right)^{1 / n}\left\|u_{h}-w\right\|_{2 ; \Omega\left(x_{0}, R\right)} .
\end{aligned}
$$

By definition of $z$

$$
\begin{aligned}
\int_{\Omega\left(x_{0}, R\right)}\left|u_{h}-w\right|^{2} d x= & \int_{\Omega\left(x_{0}, R\right)} A_{j i}^{\beta \alpha} D_{\beta} z^{j} D_{\alpha}\left(u_{h}^{i}-w^{i}\right)(1-\tau) d x \\
& +\int_{\Omega\left(x_{0}, R\right)} A_{j i}^{\beta \alpha} D_{\beta} z^{j} D_{\alpha}\left(u_{h}^{i}-w^{i}\right) \tau d x .
\end{aligned}
$$

The first term is easily estimated, since by (4.7)

$$
\begin{aligned}
& (m n)|A|_{\infty}\|D z\|_{2 ; A_{r}}\left\|D u_{h}-D w\right\|_{2 ; A_{r}} \\
& \quad \leq(m n)|A|_{\infty} c R\left(\frac{h}{R}\right)^{1 / n}\left\|u_{h}-w\right\|_{2 ; \Omega\left(x_{0}, R\right)}\left\|D u_{h}-D w\right\|_{2, \Omega\left(x_{0}, R\right)} .
\end{aligned}
$$


We rewrite the second term in view of (2.6) and (4.2) as

$$
\begin{gathered}
a\left(u_{h}-w, \tau z-\Pi_{S Z}(\tau z)\right)-\left(a-a_{0}\right)\left(w, \Pi_{S Z}(\tau z)\right)+\mathcal{F}\left(\Pi_{S Z}(\tau z)\right) \\
-\int_{\Omega\left(x_{0}, R\right)} A_{j i}^{\beta \alpha} D_{\beta} \tau z^{j} D_{\alpha}\left(u_{h}^{i}-w^{i}\right) d x .
\end{gathered}
$$

We use the interpolation inequality to estimate the first term in (4.8):

$$
\begin{aligned}
& a\left(u_{h}-w, \tau z-\Pi_{S Z}(\tau z)\right) \leq c \sum_{T \cap \operatorname{spt} \tau \neq \emptyset} h\left\|D^{2}(\tau z)\right\|_{2 ; S(T)}\left\|D u_{h}-D w\right\|_{2 ; T} \\
& \leq \varepsilon R^{2} \int_{\Omega\left(x_{0}, R\right)}\left|D u_{h}-D w\right|^{2} d x+\frac{c h^{2}}{\varepsilon R^{2}} \int_{\Omega\left(x_{0}, R\right)}\left|D^{2}(\tau z)\right|^{2} d x .
\end{aligned}
$$

By (4.6), (4.7) and Poincaré's inequality on $A_{r}$,

$$
\begin{aligned}
\int_{\Omega\left(x_{0}, R\right)}\left|D^{2}(\tau z)\right|^{2} d x & \leq c \int_{\Omega\left(x_{0}, R\right)}\left|D^{2} z\right|^{2} d x+\frac{c}{h^{2}} \int_{A_{r}}|D z|^{2} d x+\frac{c}{h^{4}} \int_{A_{r}}|z|^{2} d x \\
& \leq\left\{c+c \frac{R^{2}}{h^{2}}\left(\frac{h}{R}\right)^{2 / n}\right\} \int_{\Omega\left(x_{0}, R\right)}\left|u_{h}-w\right|^{2} .
\end{aligned}
$$

The last term in (4.8) is bounded by

$$
\begin{aligned}
& (m n)|A|_{\infty} \frac{c}{h}\|z\|_{2 ; A_{r}}\left\|D u_{h}-D w\right\|_{2 ; \Omega\left(x_{0}, R\right)} \\
& \quad \leq \varepsilon R^{2} \int_{\Omega\left(x_{0}, R\right)}\left|D u_{h}-D w\right|^{2} d x+\frac{c}{\varepsilon R^{2} h^{2}} \int_{A_{r}}|z|^{2} d x,
\end{aligned}
$$

and we proceed as before. The remaining terms in (4.8) are finally estimated with $\delta>0$ by

$$
\frac{R^{2}}{2 \delta}\left(\omega^{2}(R) \int_{\Omega\left(x_{0}, R\right)}|D w|^{2} d x+c \mathcal{R}_{h}(F, f ; R)\right)+\frac{\delta}{2} \frac{1}{R^{2}} \int_{\Omega\left(x_{0}, R\right)}\left|D \Pi_{S Z}(\tau z)\right|^{2} d x .
$$

By the stability of $\Pi_{S Z}$ (see [SZ]) we get

$$
\int_{\Omega\left(x_{0}, R\right)}\left|D \Pi_{S Z}(\tau z)\right|^{2} d x \leq c \int_{\Omega\left(x_{0}, R\right)}|D(\tau z)|^{2}+h^{2}\left|D^{2}(\tau z)\right|^{2} d x,
$$

and the estimates follow as above.

Proof of Lemma 4.1. Inequality (4.5) implies with Lemma 4.7 and Lemma 4.2 that

$$
\begin{aligned}
& \int_{\Omega\left(x_{0}, R\right)} \zeta^{2}\left|D u_{h}-D w\right|^{2} d x \\
\leq & \left\{\eta\left(\frac{h}{R}\right) \int_{\Omega\left(x_{0}, R\right)} \zeta^{2}\left|D u_{h}-\left(D u_{h}\right)_{x_{0} R}\right|^{2} d x+\omega^{2}(R) \int_{\Omega\left(x_{0}, R\right)}\left|D u_{h}\right|^{2} d x+\mathcal{R}_{h}(F, f ; R)\right\} .
\end{aligned}
$$


On the other hand, from the Campanato inequality in Proposition 3.3 for $w$ and the triangle inequality we have

$$
\begin{aligned}
\int_{\Omega\left(x_{0}, \varrho\right)}\left|D u_{h}\right|^{2} d x & \leq 2 \int_{\Omega\left(x_{0}, \varrho\right)}|D w|^{2} d x+2 \int_{\Omega\left(x_{0}, R\right)} \zeta^{2}\left|D u_{h}-D w\right|^{2} d x \\
& \leq 2 c\left(\frac{\varrho}{R}\right)^{n} \int_{\Omega\left(x_{0}, R\right)}|D w|^{2} d x+2 \int_{\Omega\left(x_{0}, R\right)} \zeta^{2}\left|D u_{h}-D w\right|^{2} d x .
\end{aligned}
$$

This yields the first inequality for $u_{h}$. The second follows analogously with the mean value form of the Campanato inequality: by the minimality of the mean value,

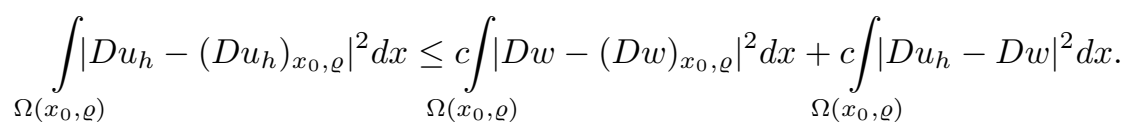

We now conclude as above. It follows from (4.3) that $(D w)_{x_{0}, R}=\left(D u_{h}\right)_{x_{0}, R}$, and we can therefore use Proposition 3.3 and Lemma 4.2 with $\xi=\left(D u_{h}\right)_{x_{0}, R}$ to estimate the first term on the right hand side. This implies the assertion of the lemma.

Proposition 4.8. Assume that $\Omega_{h}$ is a regular triangulation, $u_{h}$ the solution (2.6) and $\delta_{0}>0$.

i) Let $\lambda \in[0, n)$. Assume that $A_{i j}^{\alpha \beta} \in C^{0}(\bar{\Omega}), f \in L^{2, \lambda-2}\left(\Omega_{h}\right)$ and $F \in \mathcal{L}^{2, \lambda}\left(\Omega_{h}\right)$. Then there exist constants $R_{\lambda}^{0}, h_{\lambda}^{0}, c_{\lambda}^{0}>0$, which depend only on $\Omega, n, \lambda, A$, and $\delta_{0}$, such that $R_{\lambda}^{0}<\delta_{0}$, and for all $x_{0} \in \Omega$ with $\operatorname{dist}\left(x_{0}, \partial \Omega\right)>\delta_{0}$ and $h \leq h_{\lambda}^{0}$,

$$
\sup _{h<\varrho \leq R_{\lambda}^{0}} \frac{1}{\varrho^{\lambda}} \int_{\Omega\left(x_{0}, \varrho\right)}\left|D u_{h}\right|^{2} d x \leq c_{\lambda}^{0}\left(\left\|u_{h}\right\|_{2 ; \Omega_{h}}^{2}+\|f\|_{L^{2, \lambda-2}\left(\Omega_{h}\right)}^{2}+\|F\|_{\mathcal{L}^{2, \lambda}\left(\Omega_{h}\right)}^{2}\right) .
$$

ii) If $A_{i j}^{\alpha \beta} \in C^{0, \sigma}(\bar{\Omega})$ for some $\sigma>0, f \in L^{2, n-2}\left(\Omega_{h}\right)$ and $F \in \mathcal{L}^{2, n}\left(\Omega_{h}\right)$, then there exist constants $R_{n}^{0}, h_{n}^{0}, c_{n}^{0}>0$, which depend only on $\Omega, n, \sigma, A$, and $\delta_{0}$, such that $R_{n}^{0}<\delta_{0}$ and, for all $x_{0} \in \Omega$ with $\operatorname{dist}\left(x_{0}, \partial \Omega\right)>\delta_{0}$ and for $h \leq h_{n}^{0}$,

$$
\begin{aligned}
\sup _{h<\varrho \leq R_{n}^{0}} & \frac{1}{\varrho^{n}} \int_{\Omega\left(x_{0}, \varrho\right)}\left|D u_{h}-\left(D u_{h}\right)_{x_{0}, \varrho}\right|^{2} d x \\
& \leq c_{n}^{0}\left(\left\|u_{h}\right\|_{2 ; \Omega_{h}}^{2}+\|f\|_{L^{2, n-2}\left(\Omega_{h}\right)}^{2}+\|F\|_{\mathcal{L}^{2, n}\left(\Omega_{h}\right)}^{2}\right) .
\end{aligned}
$$

Remark. The proof shows that we only need the quantity

$$
\sup _{x_{0} \in \Omega} \sup _{h \leq R \leq R_{0}} \frac{1}{R^{\lambda}} \int_{\Omega_{h}\left(x_{0}, R\right)}\left|F-(F)_{\Omega_{h}\left(x_{0}, R\right)}\right|^{2} d x
$$

to be bounded, i.e., $F \in \mathcal{L}_{h}^{2, \lambda}\left(\Omega_{h}\right)$.

Proof. We first prove $i$ ). Let

$$
\Psi(t)=\frac{1}{t^{\lambda}} \int_{\Omega\left(x_{0}, t\right)}\left|D u_{h}\right|^{2} d x .
$$


We obtain, by Lemma 4.1 for $\varrho=\tau R$ with $\tau \in(0,1)$,

$$
\begin{aligned}
\Psi(\tau R) \leq & c\left\{\tau^{n-\lambda}+\tau^{-\lambda}\left(\omega^{2}(R)+\eta\left(\frac{h}{R}\right)\right)\right\} \Psi(R) \\
& +c \tau^{-\lambda}\left\{\|f\|_{L^{2, \lambda-2}\left(\Omega_{h}\right)}^{2}+\|F\|_{\mathcal{L}^{2, \lambda}\left(\Omega_{h}\right)}^{2}\right\},
\end{aligned}
$$

whenever $R_{0} \geq R \geq \Lambda h$ and $\tau R \geq h$, since

$$
\frac{1}{R^{\lambda}} \int_{\Omega_{h}\left(x_{0}, R\right)}\left|F-(F)_{\Omega_{h}\left(x_{0}, R\right)}\right|^{2} d x \leq c\|F\|_{L^{2, \lambda}\left(\Omega_{h}\right)}^{2}
$$

and

$$
\frac{R^{2}}{R^{\lambda}} \int_{\Omega_{h}\left(x_{0}, R\right)}|f|^{2} d x \leq c\|f\|_{L^{2, \lambda-2}\left(\Omega_{h}\right)}^{2} .
$$

Now choose first $\tau$ small enough so that $c \tau^{n-\lambda} \leq \frac{1}{4}$, then $\tilde{R}_{0}$ small enough so that $c \tau^{-\lambda} \omega\left(\tilde{R}_{0}\right) \leq \frac{1}{4}$, and finally $\Lambda_{1} \geq \max \left\{\Lambda, \tau^{-1}\right\}$ big enough so that $\eta\left(\frac{1}{\Lambda_{1}}\right) \leq \frac{1}{4}$. Let $R_{\lambda}^{0}=\min \left\{R_{0}, \tilde{R}_{0}, \delta_{0}\right\}$, and choose $h_{\lambda}^{0}$ small enough so that $\left[\Lambda_{1} h_{\lambda}^{0}, R_{\lambda}^{0}\right] \neq \emptyset$. Let $\varrho_{0} \in\left[\tau \Lambda_{1} h_{\lambda}^{0}, R_{\lambda}^{0}\right]$ be a radius such that

$$
\Psi\left(\varrho_{0}\right)=\sup _{\tau \Lambda_{1} h_{\lambda}^{0} \leq \varrho \leq R_{\lambda}^{0}} \Psi(\varrho) .
$$

If $\varrho_{0} \in\left[\tau \Lambda_{1} h_{\lambda}^{0}, \tau R_{\lambda}^{0}\right]$, then by our choice of the parameters

$$
\Psi\left(\varrho_{0}\right) \leq \frac{3}{4} \Psi\left(\tau^{-1} \varrho_{0}\right)+c \tau^{-\lambda}\left\{\|f\|_{L^{2, \lambda-2}\left(\Omega_{h}\right)}^{2}+\|F\|_{\mathcal{L}^{\lambda, n}\left(\Omega_{h}\right)}^{2}\right\},
$$

and thus

$$
\sup _{\tau \Lambda_{1} h_{\lambda}^{0} \leq \varrho \leq \tau R_{\lambda}^{0}} \Psi(\varrho) \leq 4 c \tau^{-\lambda}\left\{\|f\|_{L^{2, \lambda-2}\left(\Omega_{h}\right)}^{2}+\|F\|_{\mathcal{L}^{2, \lambda}\left(\Omega_{h}\right)}^{2}\right\} .
$$

If $\tau R_{\lambda}^{0} \leq \varrho \leq R_{\lambda}^{0}$, then

$$
\sup _{\tau R_{\lambda}^{0} \leq \varrho \leq R_{\lambda}^{0}} \Psi(\varrho) \leq \frac{1}{\left(\tau R_{\lambda}^{0}\right)^{\lambda}} \int_{\Omega\left(x_{0}, R_{\lambda}^{0}\right)}\left|D u_{h}\right|^{2} d x \leq \frac{c}{\left(\tau R_{\lambda}^{0}\right)^{\lambda}} \int_{\Omega}\left|D u_{h}\right|^{2} d x .
$$

In view of Gårding's inequality, this easily implies the assertion in case $i$ ).

To prove $i i$ ), note that $\omega^{2}(R) \leq c R^{2 \sigma}$ and therefore, in view of part $i$ ),

$$
\begin{aligned}
\frac{\omega^{2}(R)}{R^{n}} \int_{\Omega\left(x_{0}, R\right)}\left|D u_{h}\right|^{2} d x & \leq \frac{c}{R^{n-2 \sigma}} \int_{\Omega\left(x_{0}, R\right)}\left|D u_{h}\right|^{2} d x \leq c\left\|D u_{h}\right\|_{L^{2, n-2 \sigma}(\Omega)}^{2} \\
& \leq c\left\{\left\|u_{h}\right\|_{2 ; \Omega}^{2}+\|f\|_{L^{2, n-2}(\Omega)}^{2}+\|F\|_{\mathcal{L}^{2, n}(\Omega)}^{2}\right\} .
\end{aligned}
$$

The assertion of the lemma now follows as in case $i$ ) with

$$
\Psi(t)=\frac{1}{t^{n}} \int_{\Omega\left(x_{0}, t\right)}\left|D u_{h}-\left(D u_{h}\right)_{x_{0}, t}\right|^{2} d x
$$




\section{A POINTWise ESTIMATE AT THE BOUNDARY}

The estimate for $x_{0} \in \partial \Omega$ is analogous to the interior estimate in Section 4 . However, two arguments need to be modified at the boundary. First, the explicit forms of the Caccioppoli and Campanato inequalities do not seem to be directly available in the literature. We sketch the proofs in the appendix. Secondly, the interpolation operator $\Pi_{S Z}$ does not map $W_{0}^{1,2}(\Omega)$ into $W_{0}^{1,2}\left(\Omega_{h}\right)$ and must therefore be suitably modified. To do this, assume that the nodes of the triangulation are given by $a_{i}, i=1, \ldots, N$, where $a_{L+1}, \ldots, a_{N}$ are the nodes contained in $\partial \Omega_{h}$. Let $\mathcal{B}_{h}=\left\{T \in \mathcal{T}_{h}: \bar{T} \cap \partial \Omega_{h} \neq \emptyset\right\}$ and for $T \in \mathcal{B}_{h}$ define $\mathcal{N}_{0}(T)=\left\{a_{i}: a_{i} \in \bar{T} \cap \partial \Omega_{h}\right\}$. Let $\Phi_{i}$ be the standard basis in $S^{h}$.

Lemma 5.1. Assume that $\Omega$ is a domain of class $C^{1, \sigma}$ and $\Omega_{h}$ a regular triangulation. Then there exists an interpolation operator $\hat{\Pi}_{S Z}$ such that for all $v \in W_{0}^{1,2}(\Omega)$ we have $\hat{\Pi}_{S Z}(v) \in W_{0}^{1,2}\left(\Omega_{h}\right),\left.\hat{\Pi}_{S Z}(v)\right|_{T}=\left.\Pi_{S Z}(v)\right|_{T}$ for all $T \in \mathcal{T}_{h} \backslash \mathcal{B}_{h}$, and

$$
\int_{\Omega_{h}}\left|D \hat{\Pi}_{S Z}(v)-D \Pi_{S Z}(v)\right|^{2} d x \leq c h^{\sigma} \int_{\Omega \backslash \Omega_{h}}|D v|^{2} d x .
$$

Proof. This follows with a simple modification of the construction in [SZ]. Recall that $\Pi_{S Z}$ is defined by (we use the notation from [SZ])

$$
\Pi_{S Z}(v(x))=\sum_{i=1}^{N} \Phi_{i}(x) \int_{\sigma_{i}} \Psi_{i}(\xi) v(\xi) d \xi
$$

where $\sigma_{i}$ is an $(n-1)$-simplex associated with the node $a_{i}$. We define

$$
\hat{\Pi}_{S Z}(v(x))=\sum_{i=1}^{L} \Phi_{i}(x) \int_{\sigma_{i}} \Psi_{i}(\xi) v(\xi) d \xi .
$$

Clearly $\hat{\Pi}_{S Z}(v) \in W_{0}^{1,2}\left(\Omega_{h}\right)$, and $\hat{\Pi}_{S Z}$ agrees with $\Pi_{S Z}$ on all $T$ with $\mathcal{N}_{0}(T)=\emptyset$. Assume now that $\mathcal{N}_{0}(T) \neq \emptyset$. By construction, the $(n-1)$-simplices $\sigma_{i}$ associated with $a_{i} \in \mathcal{N}_{0}(T)$ are contained in $\partial \Omega_{h}$. Let $P\left(\sigma_{i}\right)=\left\{x+s \nu\left(\sigma_{i}\right): s>0, x \in \sigma_{i}\right\} \cap \Omega$, where $\nu\left(\sigma_{i}\right)$ is the outward normal to $\partial \Omega_{h}$ on $\sigma_{i}$. Since $v \in W_{0}^{1,2}(\Omega)$, we may estimate

$$
\begin{array}{r}
\int_{T}\left|D \hat{\Pi}_{S Z}(v)-D \Pi_{S Z}(v)\right|^{2} d x \leq \int_{T}\left(\sum_{a_{i} \in \mathcal{N}_{0}(T)}\left|D \Phi_{i}(x)\right| \int_{\sigma_{i}}\left|\Psi_{i}(\xi) v(\xi)\right| d \xi\right)^{2} d x \\
\leq c \sum_{a_{i} \in \mathcal{N}_{0}(T)}|T|\left|D \Phi_{i}\right|_{\infty ; T}^{2}\left|\Psi_{i}\right|_{\infty ; \sigma_{i}}^{2}\left|P\left(\sigma_{i}\right)\right| \int_{P\left(\sigma_{i}\right)}|D v|^{2} d x
\end{array}
$$

Since $\left|D \Phi_{i}\right|_{\infty ; T} \leq c h^{-1},\left|\Psi_{i}\right|_{\infty ; \sigma_{i}} \leq c h^{1-n}$, and $\operatorname{dist}(x, \partial \Omega) \leq c h^{1+\sigma}$ for all $x \in \sigma_{i}$, we obtain the assertion of the lemma.

Assume now that $x_{0} \in \partial \Omega$, and choose a domain $\Omega_{0}\left(x_{0}, R\right)$ of class $C^{2}$ such that $\Omega\left(x_{0}, R\right) \subset \Omega_{0}\left(x_{0}, R\right) \subset \Omega\left(x_{0}, 2 R\right)$. Let $\zeta$ be a smooth cut-off function such that $\zeta=\Pi_{1} \zeta=0$ on $\mathbb{R}^{n} \backslash \Omega\left(x_{0}, \frac{3}{4} R\right), \zeta=1$ on $\Omega\left(x_{0}, \frac{R}{2}\right)$, and $\|D \zeta\|_{\infty} \leq c R^{-1}$. Finally let $w \in W^{1,2}\left(\Omega_{0}\left(x_{0}, R\right)\right)$ be the solution of the system with constant coefficients $A_{i j}^{\alpha \beta}\left(x_{0}\right)$

$$
\begin{array}{rll}
a_{0}(w, \psi) & =0 & \forall \psi \in W^{1,2}\left(\Omega_{0}\left(x_{0}, R\right)\right), \\
w=u_{h} & & \text { on } \partial \Omega_{0}\left(x_{0}, R\right) .
\end{array}
$$


We need $w$ to be defined on a smooth domain, since the duality argument in Lemma 5.6 requires the solution of the adjoint problem (5.3) to be globally in $W^{2,2}$. This modification is not necessary in the scalar case, since solutions of elliptic equations in convex domains satisfy this regularity assumption. It seems to be an open question whether an analogous result holds for elliptic systems.

Lemma 5.2. Assume that $w$ is the solution of (5.1).

i) We have the a priori estimate

$$
\int_{\Omega_{0}\left(x_{0}, R\right)}|D w-\xi|^{2} d x \leq c \int_{\Omega_{0}\left(x_{0}, R\right)}\left|D u_{h}-\xi\right|^{2} d x \quad \forall \xi \in \mathbb{R}^{m n} .
$$

ii) Assume that $\Omega$ is a domain of class $C^{k}$. Then for $k \geq 2$ we have the Caccioppoli estimate

$$
\begin{aligned}
\int_{\Omega\left(x_{0}, \varrho\right)}\left|D^{k} w\right|^{2} d x \leq & \frac{c}{(R-\varrho)^{2(k-1)}} \int_{\Omega\left(x_{0}, R\right)}\left|D w-\left(D_{\nu\left(x_{0}\right)} w\right)_{x_{0}, R} \otimes \nu\left(x_{0}\right)\right|^{2} d x \\
& +\frac{c}{(R-\varrho)^{2(k-2)}} \int_{\Omega\left(x_{0}, R\right)}|D w|^{2} d x .
\end{aligned}
$$

iii) Assume that $\Omega$ is a domain of class $C^{\ell}$ with $\ell \geq k+\frac{n}{2}$. Then we have the pointwise estimate

$$
\begin{aligned}
\sup _{x \in \Omega\left(x_{0}, \varrho\right)}\left|D^{k} w\right|^{2} \leq & \frac{c}{(R-\varrho)^{2(k-1)}} \frac{1}{R^{n}} \int_{\Omega\left(x_{0}, R\right)}\left|D w-\left(D_{\nu\left(x_{0}\right)} w\right)_{x_{0}, R} \otimes \nu\left(x_{0}\right)\right|^{2} d x \\
& +\frac{c}{(R-\varrho)^{2(k-2)}} \frac{1}{R^{n}} \int_{\Omega\left(x_{0}, R\right)}|D w|^{2} d x .
\end{aligned}
$$

Proof. The proof of $i$ ) is analogous to the corresponding proof in Lemma 4.2, and we give the proof of $i i$ ) in the appendix (see Corollary A.4). Finally, iii) is a consequence of $i i$ ) and Sobolev's embedding theorem.

We define as before $\psi=\zeta^{2}\left(u_{h}-w\right)$ and $\psi_{h}=\Pi_{1} \psi$. The global estimate in Lemma $4.3 \mathrm{ii}$ ) now holds in the following form: If $\Omega$ is a domain of class $C^{k}$ with $k \geq 2+\frac{n}{2}$, then we have the global estimate

$$
\begin{aligned}
& \int_{\Omega_{h}\left(x_{0}, R\right)}\left|D \psi-D \psi_{h}\right|^{2} d x \leq \frac{c}{R^{2}} \int_{\Omega\left(x_{0}, R\right)}\left|u_{h}-w\right|^{2} d x+c R^{2}\left(\frac{h}{R}\right)^{2} \int_{\Omega\left(x_{0}, R\right)}|D w|^{2} d x \\
& +c\left(\frac{h}{R}\right)^{2} \int_{\Omega\left(x_{0}, R\right)}\left|D w-\left(D_{\nu\left(x_{0}\right)} w\right)_{x_{0}, R} \otimes \nu\left(x_{0}\right)\right|^{2} d x .
\end{aligned}
$$

We conclude from $\psi_{h} \in S_{0}^{h}$ that

$$
a\left(u_{h}-w, \psi\right)=a\left(u_{h}-w, \psi-\psi_{h}\right)+\mathcal{F}\left(\psi_{h}\right)-\left(a-a_{0}\right)\left(w, \psi_{h}\right),
$$

where we used the equations for $u_{h}$ and $w$ with $\psi_{h}$ as test function. The left hand side is estimated as in Lemma 4.4: 
Lemma 5.3. Assume that $\Omega$ is a domain of class $C^{k}$ with $k \geq 2+\frac{n}{2}$. Then

$$
a\left(u_{h}-w, \psi\right) \geq c \int_{\Omega\left(x_{0}, R\right)} \zeta^{2}\left|D u_{h}-D w\right|^{2} d x-\frac{c}{R^{2}} \int_{\Omega\left(x_{0}, R\right)}\left|u_{h}-w\right|^{2} d x .
$$

In the following lemmas we write $h^{1+\sigma}$ (instead of $h^{2}$ since $\sigma=1$ ) to indicate in which terms we use the fact that the distance to the boundary is of order $h^{1+\sigma}$.

Lemma 5.4. Assume that $\Omega$ is a domain of class $C^{k}$ with $k \geq 2+\frac{n}{2}$. Then, for $\varepsilon>0$

$$
\begin{gathered}
\left|a\left(u_{h}-w, \psi-\psi_{h}\right)\right| \leq \varepsilon \int_{\Omega\left(x_{0}, R\right)} \zeta^{2}\left|D u_{h}-D w\right|^{2} d x+c\left\{\frac{1}{\varepsilon R^{2}} \int_{\Omega\left(x_{0}, R\right)}\left|u_{h}-w\right|^{2} d x\right. \\
\left.+\frac{h}{R} \int_{\Omega\left(x_{0}, 2 R\right)}\left|D u_{h}-\left(D_{\nu\left(x_{0}\right)} u_{h}\right)_{x_{0}, 2 R} \otimes \nu\left(x_{0}\right)\right|^{2} d x+\left(\frac{h^{1+\sigma}}{R}\right)^{2 / n} \int_{\Omega_{0}\left(x_{0}, R\right)}\left|D u_{h}\right|^{2} d x\right\} .
\end{gathered}
$$

Proof. By definition

$$
a\left(u_{h}-w, \psi-\psi_{h}\right)=a_{h}\left(u_{h}-w, \psi-\psi_{h}\right)-\int_{\left(\Omega \backslash \Omega_{h}\right) \cap \operatorname{spt} \zeta} A_{i j}^{\alpha \beta} D_{\beta} w^{j} D_{\alpha}\left(\zeta^{2} w^{i}\right) d x .
$$

The first term is estimated as in Lemma 4.5, where we now use the $L^{\infty}$-estimate in Lemma $5.2 \mathrm{iii}$. To estimate the terms involving $w$, choose a rotation $Q \in \mathrm{SO}(n)$ such that $Q \nu\left(x_{0}\right)=-e_{n}$, and let $\tilde{\Omega}\left(x_{0}, R\right)=Q \Omega\left(x_{0}, R\right), \tilde{w}(x)=w\left(Q^{t} x\right)$ and $\tilde{u}_{h}(x)=u_{h}\left(Q^{t} x\right)$. Then

$$
\begin{aligned}
\int_{\Omega\left(x_{0}, R\right)}\left|D w-\left(D_{\nu\left(x_{0}\right)} w\right)_{x_{0}, R} \otimes \nu\left(x_{0}\right)\right|^{2} d x & =\int_{\tilde{\Omega}\left(x_{0}, R\right)}\left|D \tilde{w}-\left(D_{e_{n}} \tilde{w}\right)_{\tilde{\Omega}\left(x_{0}, R\right)} \otimes e_{n}\right|^{2} d x \\
& \leq \int_{\tilde{\Omega}\left(x_{0}, R\right)}\left|D \tilde{w}-\left(D_{e_{n}} \tilde{u}_{h}\right)_{\tilde{\Omega}\left(x_{0}, R\right)} \otimes e_{n}\right|^{2} d x
\end{aligned}
$$

since the mean value minimizes the integral and the resulting term can be estimated by Lemma 5.2. The second term on the right hand side is easily estimated by Hölder's inequality and Poincaré's inequality on $\left(\Omega \backslash \Omega_{h}\right) \cap \operatorname{spt} \zeta$. Finally, by the critical Sobolev embedding

$$
\begin{aligned}
\int_{\left(\Omega \backslash \Omega_{h}\right) \cap \operatorname{spt} \zeta}|D w|^{2} d x & \leq c\left(\frac{h^{1+\sigma}}{R}\right)^{2 / n}\left\{\int_{\Omega\left(x_{0}, \frac{3}{4} R\right)}|D w|^{2} d x+c R^{2} \int_{\Omega\left(x_{0}, \frac{3}{4} R\right)}\left|D^{2} w\right|^{2} d x\right\} \\
& \leq c\left(\frac{h^{1+\sigma}}{R}\right)^{2 / n} \int_{\Omega_{0}\left(x_{0}, R\right)}|D w|^{2} d x .
\end{aligned}
$$

The assertion of the lemma follows now easily by Lemma 5.2.

Recall that $\mathcal{R}_{h}$ has been defined in (4.1). 
Lemma 5.5. Assume that $\Omega$ is a domain of class $C^{k}$ with $k \geq 2+\frac{n}{2}$. Then, for $\varepsilon>0$

$$
\begin{aligned}
\left|\mathcal{F}\left(\psi_{h}\right)-\left(a-a_{0}\right)\left(w, \psi_{h}\right)\right| \leq & \frac{c}{\varepsilon}\left\{\omega^{2}(R) \int_{\Omega\left(x_{0}, R\right)}\left|D u_{h}\right|^{2} d x+\mathcal{R}_{h}(F, f ; R)\right\} \\
+ & \varepsilon\left\{\int_{\Omega\left(x_{0}, R\right)} \zeta^{2}\left|D u_{h}-D w\right|^{2} d x+\frac{1}{R^{2}} \int_{\Omega\left(x_{0}, R\right)}\left|u_{h}-w\right|^{2} d x\right. \\
& \left.+\frac{h}{R} \int_{\Omega\left(x_{0}, 2 R\right)}\left|D u_{h}-\left(D_{\nu\left(x_{0}\right)} u_{h}\right)_{x_{0}, 2 R} \otimes \nu\left(x_{0}\right)\right|^{2} d x\right\} .
\end{aligned}
$$

Proof. This is analogous to the proof of Lemma 4.6, where we now use the global estimate (5.2).

The estimate for $u_{h}-w$ is based on a duality argument as in Section 4 .

Lemma 5.6. There exists a constant $\Lambda>0$ such that for all $R \geq \Lambda$ the following inequality holds:

$$
\begin{aligned}
\int_{\Omega_{0}\left(x_{0}, R\right)}\left|u_{h}-w\right|^{2} d x & \leq \eta\left(\frac{h}{R}\right) R^{2} \int_{\Omega_{0}\left(x_{0}, R\right)}\left|D u_{h}-D w\right|^{2} d x \\
& +c R^{2}\left\{\left(\omega^{2}(R)+h^{2 \sigma / n}\right) \int_{\Omega_{0}\left(x_{0}, 2 R\right)}\left|D u_{h}\right|^{2} d x+\mathcal{R}_{h}(F, f ; 2 R)\right\} .
\end{aligned}
$$

Proof. Choose a smooth domain $\Omega_{1}\left(x_{0}, R\right)$ such that $\Omega_{1}\left(x_{0}, R\right) \subset \Omega_{0}\left(x_{0}, R\right)$ and such that there exists a cut-off function $\tau$ with the following properties: $\tau=$ $\Pi_{S Z} \tau=0$ on $\mathbb{R}^{n} \backslash \Omega_{0}\left(x_{0}, R\right), \tau=1$ on $\Omega_{1}\left(x_{0}, R\right)$, and $\left|D^{i} \tau\right| \leq c h^{-i}$ for $i=$ $0,1,2$. Moreover we may assume that $\left|\Omega_{0}\left(x_{0}, R\right) \backslash \Omega_{1}\left(x_{0}, R\right)\right| \leq \operatorname{ch} R^{n-1}$. Let $z \in W_{0}^{1,2}\left(\Omega_{0}\left(x_{0}, R\right)\right)$ be the solution of the adjoint system

$$
-D_{\alpha}\left(A_{j i}^{\beta \alpha} D_{\beta} z^{j}\right)=u_{h}^{i}-w^{i}
$$

Then $z \in W^{2,2}\left(\Omega_{0}\left(x_{0}, R\right)\right)$ (see, e.g., [Gi]) and

$$
\|z\|_{2}+R\|D z\|_{2}+R^{2}\left\|D^{2} z\right\|_{2} \leq c R^{2}\left\|u_{h}-w\right\|_{2}
$$

(the norms being taken on $\Omega_{0}\left(x_{0}, R\right)$ ). As in the proof of Lemma 4.7 with $\Pi_{S Z}$ replaced by $\hat{\Pi}_{S Z}$ we obtain

$$
\begin{aligned}
& \int_{\Omega_{0}\left(x_{0}, R\right)}\left|u_{h}-w\right|^{2} d x=\int_{\Omega_{0}\left(x_{0}, R\right)} A_{j i}^{\beta \alpha} D_{\beta} z^{j} D_{\alpha}\left(u_{h}^{i}-w^{i}\right)(1-\tau) d x \\
& \quad-\int_{\Omega_{0}\left(x_{0}, R\right)} A_{j i}^{\beta \alpha} D_{\beta} \tau z^{j} D_{\alpha}\left(u_{h}^{i}-w^{i}\right) d x-\int_{\Omega \backslash \Omega_{h}} A_{i j}^{\alpha \beta} D_{\beta} w^{j} D_{\alpha}\left(\tau z^{i}\right) d x \\
& \quad+a_{h}\left(u_{h}-w, \tau z-\hat{\Pi}_{S Z}(\tau z)\right)-\left(a-a_{0}\right)\left(w, \hat{\Pi}_{S Z}(\tau z)\right)+\mathcal{F}\left(\hat{\Pi}_{S Z}(\tau z)\right) .
\end{aligned}
$$

Denote the terms on the right hand side by $I-V I$; we estimate them separately using the inequalities

$$
\int_{\Omega_{0}\left(x_{0}, R\right) \backslash \Omega_{1}\left(x_{0}, R\right)}|D(\tau z)|^{2} d x \leq c R^{2}\left(\frac{h}{R}\right)^{2 / n} \int_{\Omega_{0}\left(x_{0}, R\right)}\left|u_{h}-w\right|^{2} d x
$$


and

$$
\int_{\Omega \backslash \Omega_{h}}|D(\tau z)|^{2} d x \leq c R^{2}\left(\frac{h^{1+\sigma}}{R}\right)^{2 / n} \int_{\Omega_{0}\left(x_{0}, R\right)}\left|u_{h}-w\right|^{2} d x
$$

which follow from Poincaré's inequality, the Sobolev embedding and the a priori estimate (5.4). Now

$$
|I| \leq(m n)|A|_{\infty}\left(\int_{\Omega_{0}\left(x_{0}, R\right) \backslash \Omega_{1}\left(x_{0}, R\right)}|D z|^{2} d x\right)^{1 / 2}\left(\int_{\Omega_{0}\left(x_{0}, R\right)}\left|D u_{h}-D w\right|^{2} d x\right)^{1 / 2}
$$

and

$$
|I I| \leq(m n)|A|_{\infty}|D \tau|_{\infty}\left(\int_{\operatorname{spt} D \tau}|z|^{2} d x\right)^{1 / 2}\left(\int_{\Omega_{0}\left(x_{0}, R\right)}\left|D u_{h}-D w\right|^{2} d x\right)^{1 / 2}
$$

are easily estimated. For $I I I$ we obtain

$$
|I I I| \leq c(m n)|A|_{\infty}\left(\int_{\Omega_{0}\left(x_{0}, R\right)}|D w|^{2} d x\right)^{1 / 2} R\left(\frac{h^{1+\sigma}}{R}\right)^{1 / n}\left(\int_{\Omega_{0}\left(x_{0}, R\right)}\left|u_{h}-w\right|^{2} d x\right)^{1 / 2},
$$

and this can be estimated by Young's inequality. To bound the remaining terms $I V-V I$ we use the fact that by Lemma 5.1 and (5.6)

$$
\begin{aligned}
\int_{\Omega_{h}}\left|\Pi_{S Z}(\tau z)-\hat{\Pi}_{S Z}(\tau z)\right|^{2} d x & \leq c h^{\sigma} \int_{\Omega \backslash \Omega_{h}}|D(\tau z)|^{2} d x \\
& \leq c h^{\sigma(1+2 / n)} R^{2}\left(\frac{h}{R}\right)^{2 / n} \int_{\Omega_{0}\left(x_{0}, R\right)}\left|u_{h}-w\right|^{2} d x .
\end{aligned}
$$

The assertion follows easily.

The above lemmas prove the following Campanato inequality for $u_{h}$ at the boundary. The proof is identical to the proof of Lemma 4.1.

Lemma 5.7. Assume that $\Omega$ is a domain of class $C^{k}$ with $k \geq 2+\frac{n}{2}$. Then there exists a constant $\Lambda>0$ such that for all $h \leq \varrho \leq R \leq R_{0}$ and $R \geq \Lambda h$ the following inequalities hold:

$$
\begin{aligned}
& \int_{\Omega\left(x_{0}, \varrho\right)}\left|D u_{h}\right|^{2} d x \\
& \quad \leq c\left\{\left(\frac{\varrho}{R}\right)^{n}+\omega^{2}(R)+\eta\left(\frac{h}{R}\right)+h^{2 \sigma / n}\right\} \int_{\Omega\left(x_{0}, 2 R\right)}\left|D u_{h}\right|^{2} d x+c \mathcal{R}_{h}(F, f ; 2 R),
\end{aligned}
$$




$$
\begin{aligned}
& \int_{\Omega\left(x_{0}, \varrho\right)}\left|D u_{h}-\left(D_{\nu\left(x_{0}\right)} u_{h}\right)_{x_{0}, \varrho} \otimes \nu\left(x_{0}\right)\right|^{2} d x \\
& \leq c\left\{\left(\frac{\varrho}{R}\right)^{n+2}+\eta\left(\frac{h}{R}\right)\right\} \int_{\Omega\left(x_{0}, 2 R\right)}\left|D u_{h}-\left(D_{\nu\left(x_{0}\right)} u_{h}\right)_{x_{0}, R} \otimes \nu\left(x_{0}\right)\right|^{2} d x \\
&+c\left(\omega^{2}(R)+h^{2 \sigma / n}\right) \int_{\Omega\left(x_{0}, 2 R\right)}\left|D u_{h}\right|^{2} d x+c \mathcal{R}_{h}(F, f ; 2 R) .
\end{aligned}
$$

Here $\Lambda$ is independent of $x_{0}, h, \varrho, R, u, u_{h}$, while $\eta$ is a nonnegative, continuous function such that $\eta(t) \leq c t^{1 / n}$ for $n \geq 3$ and $\eta(t) \leq c(\mu) t^{\mu}$ for all $\mu \in\left(0, \frac{1}{2}\right)$ for $n=2$.

We obtain from this Campanato inequality the following estimate at the boundary:

Proposition 5.8. Assume that $\Omega$ is a domain of class $C^{k}$ with $k \geq 2+\frac{n}{2}, \Omega_{h}$ a regular triangulation and $u_{h}$ the solution of (2.6). Let $x_{0} \in \partial \Omega$.

i) Let $\lambda \in[0, n)$. Assume that $A_{i j}^{\alpha \beta} \in C^{0}(\bar{\Omega}), f \in L^{2, \lambda-2}\left(\Omega_{h}\right)$ and $F \in \mathcal{L}^{2, \lambda}\left(\Omega_{h}\right)$. Then there exist constants $R_{\lambda}^{1}, h_{\lambda}^{1}, c_{\lambda}^{1}>0$, which depend only on $\Omega, n, \lambda$, and $A$, such that for $h \leq h_{\lambda}^{1}$

$$
\sup _{h<\varrho \leq R_{\lambda}^{1}} \frac{1}{\varrho^{\lambda}} \int_{\Omega\left(x_{0}, \varrho\right)}\left|D u_{h}\right|^{2} d x \leq c_{\lambda}^{1}\left(\left\|u_{h}\right\|_{2 ; \Omega_{h}}^{2}+\|f\|_{L^{2, \lambda-2}\left(\Omega_{h}\right)}^{2}+\|F\|_{\mathcal{L}^{2, \lambda}\left(\Omega_{h}\right)}^{2}\right) .
$$

ii) If $A_{i j}^{\alpha \beta} \in C^{0, \sigma}(\bar{\Omega})$ for some $\sigma>0, f \in L^{2, n-2}\left(\Omega_{h}\right)$ and $F \in \mathcal{L}^{2, n}\left(\Omega_{h}\right)$, then there exist constants $R_{n}^{1}, h_{n}^{1}, c_{n}^{1}>0$, which depend only on $\Omega, n, \sigma$, and $A$, such that for $h \leq h_{n}^{1}$

$$
\begin{aligned}
\sup _{h<\varrho \leq R_{n}^{1}} & \frac{1}{\varrho^{n}} \int_{\Omega\left(x_{0}, \varrho\right)}\left|D u_{h}-\left(D u_{h}\right)_{x_{0}, \varrho}\right|^{2} d x \\
& \leq c_{n}^{1}\left(\left\|u_{h}\right\|_{2 ; \Omega_{h}}^{2}+\|f\|_{L^{2, n-2}\left(\Omega_{h}\right)}^{2}+\|F\|_{\mathcal{L}^{2, n}\left(\Omega_{h}\right)}^{2}\right) .
\end{aligned}
$$

Proof. The proof is analogous to the proof of Proposition 4.8. In the proof of $i$ ) we choose $h_{\lambda}^{1}$ small enough so that $c\left(h_{\lambda}^{1}\right)^{2 / n} \leq \frac{1}{8}$, while in the proof of $\left.i i\right)$ we use the inequality $h \leq R$. Thus we obtain

$$
\begin{aligned}
& \sup _{h<\varrho \leq R_{n}^{1}} \frac{1}{\varrho^{n}} \int_{\Omega\left(x_{0}, \varrho\right)}\left|D u_{h}-\left(D_{\nu\left(x_{0}\right)} u_{h}\right)_{x_{0}, \varrho} \otimes \nu\left(x_{0}\right)\right|^{2} d x \\
& \quad \leq c_{n}^{1}\left(\left\|u_{h}\right\|_{2 ; \Omega_{h}}^{2}+\|f\|_{L^{2, n-2}\left(\Omega_{h}\right)}^{2}+\|F\|_{\mathcal{L}^{2, n}\left(\Omega_{h}\right)}^{2}\right) .
\end{aligned}
$$

The proposition follows since the mean value minimizes the integral on the left hand side.

\section{Error estimates in Campanato spaces}

The estimates in Sections $4-5$ imply the following stability result.

Theorem 6.1. Assume that $\Omega$ is a domain of class $C^{k}$ with $k \geq 2+\frac{n}{2}, \Omega_{h} a$ regular triangulation and $u_{h}$ a solution of (2.6). 
i) Let $\lambda \in[0, n)$. Assume that $A_{i j}^{\alpha \beta} \in C^{0}(\bar{\Omega}), f \in L^{2, \lambda-2}\left(\Omega_{h}\right)$ and $F \in \mathcal{L}^{2, \lambda}\left(\Omega_{h}\right)$. Then there exists a constant $c_{\lambda}>0$, which depends only on $\Omega, n, \lambda$, and $A$, such that

$$
\left\|D u_{h}\right\|_{L^{2, \lambda}\left(\Omega_{h}\right)} \leq c_{\lambda}\left(\left\|u_{h}\right\|_{2 ; \Omega_{h}}+\|f\|_{L^{2, \lambda-2}\left(\Omega_{h}\right)}+\|F\|_{\mathcal{L}^{2, \lambda}\left(\Omega_{h}\right)}\right) .
$$

ii) If $A_{i j}^{\alpha \beta} \in C^{0, \sigma}(\bar{\Omega})$ for some $\sigma>0, f \in L^{2, n-2}\left(\Omega_{h}\right)$ and $F \in \mathcal{L}^{2, n}\left(\Omega_{h}\right)$, then there exists a constant $c_{n}>0$, which depends only on $\Omega, n, \sigma$, and $A$, such that

$$
\left\|D u_{h}\right\|_{\mathcal{L}^{2, n}\left(\Omega_{h}\right)} \leq c_{n}\left(\left\|u_{h}\right\|_{2 ; \Omega_{h}}+\|f\|_{L^{2, n-2}\left(\Omega_{h}\right)}+\|F\|_{\mathcal{L}^{2, n}\left(\Omega_{h}\right)}\right) .
$$

iii) If the system (2.3) has a unique solution, then the estimates in i) and ii) hold without the norm of $u_{h}$ on the right hand side.

iv) If the system has a unique solution, then the Ritz projection is stable in Morrey and Campanato spaces: under the assumptions in i) and ii) we have

$$
\left\|D u_{h}\right\|_{L^{2, \lambda}\left(\Omega_{h}\right)} \leq c_{\lambda}\|D u\|_{L^{2, \lambda}\left(\Omega_{h}\right)}
$$

and

$$
\left\|D u_{h}\right\|_{\mathcal{L}^{2, n}\left(\Omega_{h}\right)} \leq c_{n}\|D u\|_{\mathcal{L}^{2, n}\left(\Omega_{h}\right)} .
$$

Proof. To prove $i$ ), let $\bar{R}=\min \left\{R_{\lambda}^{0}, \frac{1}{2} R_{\lambda}^{1}\right\}$ and assume that $h \leq \min \left\{h_{\lambda}^{0}, h_{\lambda}^{1}\right\}$. We first show that

$$
\sup _{x_{0} \in \bar{\Omega}} \sup _{h \leq R \leq \bar{R}} \frac{1}{R^{\lambda}} \int_{\Omega\left(x_{0}, R\right)}\left|D u_{h}\right|^{2} d x \leq c\left(\left\|u_{h}\right\|_{2 ; \Omega}^{2}+\|f\|_{L^{2, \lambda-2}\left(\Omega_{h}\right)}^{2}+\|F\|_{\mathcal{L}^{2, \lambda}\left(\Omega_{h}\right)}^{2}\right) .
$$

This inequality follows from the interior estimate in Proposition 4.8 and the estimate at the boundary if $\Omega\left(x_{0}, R\right) \subset \Omega$ or $x_{0} \in \partial \Omega$, respectively. Assume now that $\Omega\left(x_{0}, R\right) \cap \partial \Omega \neq \emptyset$ and $x_{0} \notin \partial \Omega$. Choose a point $\bar{x}_{0} \in \partial \Omega$ such that $\left|x_{0}-\bar{x}_{0}\right|=$ $\operatorname{dist}\left(x_{0}, \partial \Omega\right)$. Since

$$
\frac{1}{R^{\lambda}} \int_{\Omega\left(x_{0}, R\right)}\left|D u_{h}\right|^{2} d x \leq \frac{c}{(2 R)^{\lambda}} \int_{\Omega\left(\bar{x}_{0}, 2 R\right)}\left|D u_{h}\right|^{2} d x
$$

we conclude this proof using again the boundary estimate in Proposition 4.8. The assertion of case $i$ ) now follows easily from Lemma 3.4. The proof of $i i$ ) is analogous, and iii) follows from Theorem 3.1. To prove $i v$ ), define $F_{i}^{\alpha}=A_{i j}^{\alpha \beta} D_{\beta} u^{j}$ and note that $F \in L^{2, \lambda}(\Omega)\left(F \in \mathcal{L}^{2, n}(\Omega)\right)$ if $D u \in L^{2, \lambda}(\Omega)\left(D u \in \mathcal{L}^{2, n}(\Omega)\right)$. This follows from the fact that $L^{\infty}(\Omega)$ and $C^{0, \sigma}(\bar{\Omega})$ are multipliers in $L^{2, \lambda}(\Omega)$ and $\mathcal{L}^{2, n}(\Omega)$, respectively. The assertion is now an immediate consequence of the a priori estimates in $i$ ) and $i i)$.

The following optimal error estimates are an immediate consequence of Theorem 6.1 .

Theorem 6.2. Assume that $\Omega$ is a domain of class $C^{k}$ with $k \geq 2+\frac{n}{2}, \Omega_{h} a$ regular triangulation, and $u$ and $u_{h}$ the unique solutions of the system (2.5) and the finite element equation (2.6), respectively. Define $e_{h}=u-u_{h}$. 
i) Let $\lambda \in[0, n)$. Assume that $A_{i j}^{\alpha \beta} \in C^{0}(\bar{\Omega}), f \in L^{2, \lambda-2}\left(\Omega_{h}\right)$ and $F \in \mathcal{L}^{2, \lambda}\left(\Omega_{h}\right)$. Then there exists a constant $c_{\lambda}>0$, which depends only on $\Omega, n, \lambda$, and $A$, such that

$$
\left\|D e_{h}\right\|_{L^{2, \lambda}\left(\Omega_{h}\right)} \leq c_{\lambda} \inf _{w_{h} \in S_{0}^{h}}\left\|D u-D w_{h}\right\|_{L^{2, \lambda}\left(\Omega_{h}\right)}
$$

ii) Let $\lambda \in(n-2, n)$. Assume that $A_{i j}^{\alpha \beta} \in C^{1}(\bar{\Omega}), f \in L^{2, \lambda}\left(\Omega_{h}\right)$ and $F \in W^{1,2}(\Omega)$ with $D F \in \mathcal{L}^{2, \lambda}\left(\Omega_{h}\right)$. Then there exists a constant $c_{\lambda}>0$, which depends only on $\Omega, n, \lambda$, and $A$, such that we have the optimal estimate

$$
\left\|D e_{h}\right\|_{L^{2, \lambda}\left(\Omega_{h}\right)} \leq c_{\lambda} h\left\|D^{2} u\right\|_{L^{2, \lambda}\left(\Omega_{h}\right)}
$$

iii) If $A_{i j}^{\alpha \beta} \in C^{0, \sigma}(\bar{\Omega})$ for some $\sigma>0, f \in L^{2, n-2}\left(\Omega_{h}\right)$ and $F \in \mathcal{L}^{2, n}\left(\Omega_{h}\right)$, then there exists a constant $c_{n}>0$, which depends only on $\Omega, n, \sigma$, and $A$, such that

$$
\left\|D e_{h}\right\|_{\mathcal{L}^{2, n}\left(\Omega_{h}\right)} \leq c_{n} \inf _{w_{h} \in S_{0}^{h}}\left\|D u-D w_{h}\right\|_{\mathcal{L}^{2, n}\left(\Omega_{h}\right)}
$$

iv) If $A_{i j}^{\alpha \beta} \in C^{1, \sigma}(\bar{\Omega})$ for some $\sigma>0, f \in L^{2, \lambda}\left(\Omega_{h}\right)$ for some $\lambda \in(n, n+2]$ and $F \in W^{1,2}(\Omega)$ with $D F \in \mathcal{L}^{2, \lambda}\left(\Omega_{h}\right)$, then there exists a constant $c_{n}>0$, which depends only on $\Omega, n, \sigma$, and $A$, such that

$$
\left\|D e_{h}\right\|_{\mathcal{L}^{2, n}\left(\Omega_{h}\right)} \leq c_{n} h\left\|D^{2} u\right\|_{\infty ; \Omega_{h}} .
$$

Remarks. 1) The system (2.3) has for exampe a unique solution if the coefficients are constant or if the coefficients $A_{i j}^{\alpha \beta}$ satisfy the Legendre condition, i.e., there exists a constant $c>0$ such that $A_{i j}^{\alpha \beta}(x) \xi_{\alpha}^{i} \xi_{\beta}^{j} \geq c|\xi|^{2}$ for all $\xi \in \mathbb{R}^{m n}$ and all $x \in \bar{\Omega}$.

2) The slightly stronger assumptions in part $i v$ ) of Theorem 6.2 compared with Theorem $6.1 \mathrm{ii}$ ) are needed in order to ensure that $D^{2} u \in L^{\infty}$. In fact, $D^{2} u \in C^{0, \sigma}$ with $\sigma=\frac{\lambda-n}{2}$; see Section 3 .

Proof. We first prove $i)$. For $w_{h} \in S_{0}^{h}$ define $F_{i}^{\alpha}=A_{i j}^{\alpha \beta} D_{\beta}\left(u^{j}-w_{h}^{j}\right)$, and let $v_{h}$ be the finite element solution of $(2.3)$ with $F=\left(F_{i}^{\alpha}\right)$ and $f=0$. By Theorem 6.1

$$
\left\|D v_{h}\right\|_{L^{2, \lambda}\left(\Omega_{h}\right)} \leq c\left\|D u-D w_{h}\right\|_{L^{2, \lambda}\left(\Omega_{h}\right)} .
$$

Since the solutions of the system (2.3) are unique, $v_{h}=\mathcal{R}\left(u-w_{h}\right)$ is the Ritz projection of $u-w_{h}$, and this estimate implies

$$
\left\|D u_{h}-D w_{h}\right\|_{L^{2, \lambda}\left(\Omega_{h}\right)} \leq c\left\|D u-D w_{h}\right\|_{L_{h}^{2, \lambda}\left(\Omega_{h}\right)} .
$$

The proof follows now from the triangle inequality:

$$
\left\|D u-D u_{h}\right\|_{L^{2, \lambda}\left(\Omega_{h}\right)} \leq\left\|D u-D w_{h}\right\|_{L^{2, \lambda}\left(\Omega_{h}\right)}+\left\|D u_{h}-D w_{h}\right\|_{L^{2, \lambda}\left(\Omega_{h}\right)} .
$$

To prove $i i)$, we have to show that for $\lambda \in(n-2, n)$ the estimate

$$
\left\|D u-D \Pi_{S Z} u\right\|_{L^{2, \lambda}\left(\Omega_{h}\right)} \leq \operatorname{ch}\left\|D^{2} u\right\|_{L^{2, \lambda}\left(\Omega_{h}\right)}
$$


holds. In view of Poincaré's inequality, we deduce that $D u \in \mathcal{L}^{2, \lambda+2}$, and thus $D u \in C^{0, \sigma}(\bar{\Omega})$ with $\sigma=\frac{\lambda+2-n}{2}$. Hence for $0<\varrho<h$ we obtain

$$
\begin{aligned}
& \frac{1}{\varrho^{\lambda}} \int_{\Omega_{h}\left(x_{0}, \varrho\right)}\left|D u-D \Pi_{S Z} u\right|^{2} d x \\
& \quad \leq \frac{c}{\varrho^{\lambda}} \int_{\Omega_{h}\left(x_{0}, \varrho\right)}\left|D u-D u\left(x_{0}\right)\right|^{2} d x+\frac{c}{\varrho^{\lambda}} \int_{\Omega_{h}\left(x_{0}, \varrho\right)}\left|D \Pi_{S Z} u-D u\left(x_{0}\right)\right|^{2} d x .
\end{aligned}
$$

With $\bar{x}_{0}$ and $\varrho$ defined as in Lemma 3.4, we conclude that

$$
\begin{aligned}
& \frac{c}{\varrho^{\lambda}} \int_{\Omega_{h}\left(x_{0}, \varrho\right)}\left|D \Pi_{S Z} u-D u\left(x_{0}\right)\right|^{2} d x \\
& \quad \leq \frac{c}{\bar{\varrho}^{\lambda}} \int_{\Omega_{h}\left(\bar{x}_{0}, \bar{\varrho}\right)}\left|D \Pi_{S Z} u-D u\right|^{2} d x+\frac{c}{\bar{\varrho}^{\lambda}} \int_{\Omega_{h}\left(\bar{x}_{0}, \bar{\varrho}\right)}\left|D u-D u\left(x_{0}\right)\right|^{2} d x .
\end{aligned}
$$

Since $D u \in C^{0, \sigma}(\bar{\Omega})$, we get

$$
\frac{c}{\varrho^{\lambda}} \int_{\Omega_{h}\left(x_{0}, \varrho\right)}\left|D u-D u\left(x_{0}\right)\right|^{2} d x \leq c \varrho^{n-\lambda+2 \sigma}\left\|D^{2} u\right\|_{L^{2, \lambda}\left(\Omega_{h}\right)}^{2} \leq c h^{2}\left\|D^{2} u\right\|_{L^{2, \lambda}\left(\Omega_{h}\right)}^{2}
$$

and a similar inequality for the integral over $\Omega\left(\bar{x}_{0}, \bar{\varrho}\right)$. This proves $\left.i i\right)$, since we may use the interpolation property of $\Pi_{S Z}$ on balls with radii of order $h$. The proof of iii) is similar. Finally, $i v)$ follows since $u \in W^{2, \infty}(\Omega)$ implies

$$
\sup _{x \in \bar{\Omega}} \sup _{0<R \leq \operatorname{diam}(\Omega)} \frac{1}{R^{n}} \int_{\Omega_{h}\left(x_{0}, R\right)}\left|D u-D \Pi_{1} u\right|^{2} d x \leq c h^{2}\left\|D^{2} u\right\|_{\infty ; \Omega_{h}}^{2} .
$$

\section{UNIFORM ESTIMATES}

As a further application of the $\mathcal{L}^{2, n}$ estimates in Theorem 6.2 we show in this section how one can deduce from these estimates an optimal $L^{\infty}$ estimate. This generalizes the famous results in [RS] to systems in arbitrary dimensions. The case of an elliptic equation in arbitrary dimensions has recently been solved in [SW2]. The methods employed in this section were first used in [DF].

Theorem 7.1. Assume that $\Omega$ is a domain of class $C^{k}$ with $k \geq 2+\frac{n}{2}, \Omega_{h}$ a regular triangulation, and that $u$ and $u_{h}$ are the unique solutions of the system (2.5) and the finite element equation (2.6), respectively. Define $e_{h}=u-u_{h}$. If $A_{i j}^{\alpha \beta} \in C^{2, \sigma}(\bar{\Omega})$ for some $\sigma>0, f \in L^{2, \lambda}\left(\Omega_{h}\right)$, and $F \in W^{1,2}(\Omega)$ is such that $D F \in \mathcal{L}^{2, \lambda}\left(\Omega_{h}\right)$ with $\lambda \in(n, n+2]$, then

$$
\left\|D e_{h}\right\|_{\infty ; \Omega_{0}} \leq \operatorname{ch}\left\|D^{2} u\right\|_{\infty ; \Omega}
$$

for all $\Omega_{0} \subset \subset \Omega$.

Proof. We restrict ourselves to the case of interior estimates for systems with constant coefficients; the proof with $C^{2, \sigma}$ coefficients is similar, since the corresponding Green's function has the same growth properties, see $[F],[D M]$. We give the arguments for $n \geq 3$; for $n=2$ one uses the logarithmic Green's function. The key 
point in the argument is to use a differentiated Green's function as introduced in [RS].

Assume that $\left\|D e_{h}\right\|_{\infty ; \Omega_{0}}=D_{s} e_{h}^{k}\left(x_{0}\right)$, where $x_{0} \in T_{0} \in \mathcal{T}_{h}$. Choose $\hat{x}_{0} \in T_{0}$ such that $\Omega\left(\hat{x}_{0}, \sigma_{0} h\right) \subset T_{0}$, and let $\tilde{e}_{h}=\Pi_{1} u-u_{h}$. In view of the interpolation estimate,

$$
\left|D_{s} e_{h}^{k}\left(x_{0}\right)\right| \leq\left|D_{s} \tilde{e}_{h}^{k}\left(x_{0}\right)\right|+\left|D_{s}\left(u^{k}-\Pi_{1} u^{k}\right)\left(x_{0}\right)\right| \leq\left|D_{s} \tilde{e}_{h}^{k}\left(\hat{x}_{0}\right)\right|+\mathcal{O}(h),
$$

and thus it suffices to estimate $D_{s} \tilde{e}_{h}^{k}\left(\hat{x}_{0}\right)$. Choose a smooth function $\delta \geq 0$ with support in $\Omega\left(\hat{x}_{0}, \sigma_{0} / 2\right)$ and $\int_{\mathbb{R}^{n}} \delta d x=1$. Denote by $G=G(x, y) \in W_{0}^{1,2}(\Omega)$ the solution of the elliptic system

$$
-D_{\alpha}\left(A_{j i}^{\beta \alpha} D_{\beta} G^{j}\right)=-\delta_{i k} \delta_{h}
$$

where $\delta_{h}=h^{-n} \delta\left(\frac{x-y}{h}\right)$. Taking the derivative with respect to $x_{s}$ we obtain a solution $G_{s}=D_{s} G$ of the system

$$
-D_{\alpha}\left(A_{j i}^{\beta \alpha} D_{\beta} G_{s}^{j}\right)=-\delta_{i k} D_{s} \delta_{h} .
$$

With $y=\hat{x}_{0}$ we deduce by standard $L^{2}$ estimates that

$$
\begin{gathered}
\int_{\Omega\left(\hat{x}_{0}, \sigma h\right)}\left|D G_{s}\right|^{2} d x \leq c_{\sigma} h^{-n} \\
\int_{\Omega\left(\hat{x}_{0}, \sigma h\right)}\left|D^{2} G_{s}\right|^{2} d x \leq c_{\sigma} h^{-n-2}
\end{gathered}
$$

for $\sigma \leq \sigma_{0}$, and a slight generalization of the estimates for elliptic systems in [F] (see also $[\mathrm{DM}]$ ) shows that the following pointwise estimates hold on $\Omega \backslash \Omega\left(\hat{x}_{0}, \sigma_{0} h\right)$ :

$$
\begin{aligned}
\left|G_{s}\right| & \leq c\left|x-\hat{x}_{0}\right|^{1-n} \\
\left|D G_{s}\right| & \leq c\left|x-\hat{x}_{0}\right|^{-n} \\
\left|D^{2} G_{s}\right| & \leq c\left|x-\hat{x}_{0}\right|^{-n-1} .
\end{aligned}
$$

Fix $0<R_{0}<\operatorname{dist}\left(\Omega_{0}, \partial \Omega\right) / 2$, and choose a cut-off function $\tau_{0}$ such that $\tau_{0}=1$ on $\Omega\left(x_{0}, R_{0} / 2\right), \tau_{0}=\Pi_{S Z} \tau_{0}=0$ on $\mathbb{R}^{n} \backslash \Omega\left(x_{0}, R_{0}\right)$ and $\left|D^{i} \tau_{0}\right| \leq c R_{0}^{-i}$ for $i=1,2$. Then

$$
\begin{aligned}
D_{s} \tilde{e}_{h}^{k}\left(\hat{x}_{0}\right) & =a_{h}\left(\tau_{0} \tilde{e}_{h}, G_{s}\right)=a_{h}\left(e_{h}, \tau_{0} G_{s}\right)+a_{h}\left(\tau_{0}\left(\Pi_{1} u-u\right), G_{s}\right) \\
& +\int_{\Omega_{h}} A_{j i}^{\beta \alpha} D_{\beta} G_{s}^{j}\left(D_{\alpha} \tau_{0}\right) e_{h}^{i} d x-\int_{\Omega_{h}} A_{j i}^{\beta \alpha}\left(D_{\beta} \tau_{0}\right) G_{s}^{j} D_{\alpha} e_{h}^{i} d x .
\end{aligned}
$$

Using integration by parts and the estimates for $G_{s}$, we see that the second term on the right hand side in (7.1) is of order $\mathcal{O}(h)$. It is here that we use the full strength of the differentiated Green's function. Since $\tau_{0}=1$ on $\Omega\left(\hat{x}_{0}, R_{0} / 2\right)$, the two integrals involving $D \tau_{0}$ in (7.1) are estimated in view of the $W^{1,2}$ estimates for $e_{h}$. Let $\psi=\tau_{0} G_{s}$ and $\psi_{h}=\Pi_{S Z} \psi$. By the orthogonality of the Ritz projection we conclude from (7.1) that

$$
D_{s} \tilde{e}_{h}^{k}\left(\hat{x}_{0}\right)=\int_{\Omega_{h}} A_{i j}^{\alpha \beta} D_{\beta} e_{h}^{j} D_{\alpha}\left(\psi^{i}-\psi_{h}^{i}\right) d x+\mathcal{O}(h) .
$$

To estimate the remaining integral, we define a family of balls $\Omega\left(\hat{x}_{0}, R_{\ell}\right)$ with $R_{\ell}=$ $2^{-\ell} R_{0}, \ell=-1,0,1, \ldots, L$, such that $R_{L} \leq h<R_{L-1}$, and corresponding cut-off functions $\tau_{\ell}$ such that $\sum \tau_{\ell}=1$ on $\Omega\left(\hat{x}_{0}, R_{0}\right)$ and

$$
\operatorname{spt}\left(\tau_{\ell}\right) \subset \Omega\left(\hat{x}_{0}, R_{\ell-1}\right) \backslash \Omega\left(\hat{x}_{0}, R_{\ell+1}\right) \text {. }
$$


Then

$$
\begin{aligned}
\int_{\Omega_{h}} A_{i j}^{\alpha \beta} & D_{\beta} e_{h}^{j} D_{\alpha}\left(\psi^{i}-\psi_{h}^{i}\right) d x \\
& =\sum_{\ell=-1}^{L} \int_{\Omega_{h}} A_{i j}^{\alpha \beta}\left(D_{\beta} e_{h}^{j}-\left(D_{\beta} e_{h}^{j}\right)_{\hat{x}_{0}, R_{\ell}}\right) D_{\alpha}\left(\tau_{\ell}\left(\psi^{i}-\psi_{h}^{i}\right)\right) d x .
\end{aligned}
$$

In view of the $\mathcal{L}^{2, n}$-estimate for $D e_{h}$ this is estimated by

$$
c \sum_{\ell=-1}^{L} h R_{\ell}^{n / 2}\left\|D_{\alpha}\left(\tau_{\ell}\left(\psi^{i}-\psi_{h}^{i}\right)\right)\right\|_{2 ; \Omega\left(\hat{x}_{0}, R_{\ell}\right)} .
$$

Invoking again the estimates for $G_{s}$, we obtain

$$
\left\|D e_{h}\right\|_{\infty ; \Omega_{0}} \leq c h^{2}\left\|D^{2} u\right\|_{\infty ; \Omega} \sum_{\ell=-1}^{L} R_{\ell}^{-1}+\mathcal{O}(h)=\mathcal{O}(h),
$$

and this proves the assertion of the theorem.

\section{ApPendix}

The following lemmas contain estimates for solutions of elliptic systems which do not seem to be directly available in the literature. The proofs use standard techniques and are included for the convenience of the reader.

Lemma A.1. Assume that $A_{i j}^{\alpha \beta} \in C^{1}\left(B^{+}(0,2 R)\right)$ and that $v \in C^{2}\left(B^{+}(0,2 R)\right)$ is a solution of

$$
D_{\alpha}\left(A_{i j}^{\alpha \beta} D_{\beta} v^{j}\right)=0
$$

with $v=0$ on $\partial B^{+}(0,2 R) \cap\left\{x_{n}=0\right\}$. Then

$$
\int_{B^{+}(0, R)}\left|D^{2} v\right|^{2} d x \leq \frac{c}{R^{2}} \int_{B^{+}(0,2 R)}\left|D v-\xi \otimes e_{n}\right|^{2} d x+c R^{n}|\xi|^{2}
$$

for all $\xi \in \mathbb{R}^{m}$.

Proof. Let $\zeta \in C_{0}^{\infty}\left(\mathbb{R}^{n}\right)$ be a cut-off function such that $\zeta \equiv 1$ on $B(0, R), \zeta \equiv 0$ on $\mathbb{R}^{n} \backslash B(0,2 R)$ and $|D \zeta| \leq c R^{-1}$ with a constant independent of $R$. Define $w=\zeta\left(v-x_{n} \xi\right)$. Then $D_{\beta} w=\zeta\left(D_{\beta} v-\delta_{\beta n} \xi\right)+D_{\beta} \zeta\left(v-x_{n} \xi\right)$ and

$$
\begin{aligned}
& D_{\alpha}\left(A_{i j}^{\alpha \beta} D_{\beta} w^{j}\right) \\
& =(-\zeta) D_{\alpha} A_{i j}^{\alpha \beta} \delta_{\beta n} \xi^{j}+D_{\alpha} A_{i j}^{\alpha \beta}\left(D_{\beta} v^{j}-\delta_{\beta n} \xi^{j}\right)+D_{\alpha}\left(A_{i j}^{\alpha \beta} D_{\beta} \zeta\left(v^{j}-x_{n} \xi\right)\right) \\
& =f_{i}+D_{\alpha} F_{i}^{\alpha}
\end{aligned}
$$

with

$$
f_{i}=(-\zeta) D_{\alpha} A_{i j}^{\alpha \beta} \delta_{\beta n} \xi^{j}+\left(D_{\alpha} \zeta^{j}\right) A_{i j}^{\alpha \beta}\left(D_{\beta} v^{j}-\delta_{\beta n} \xi^{j}\right)
$$

and

$$
F_{i}^{\alpha}=A_{i j}^{\alpha \beta} D_{\beta} \zeta\left(v^{j}-x_{n} \xi\right)
$$

It follows from standard results in elliptic regularity (see, e.g., [Gi], p. 363) that

$$
\int_{B^{+}(0, R)}\left|D^{2} w\right|^{2} d x \leq \frac{c}{R^{2}} \int_{B^{+}(0,2 R)}|D w|^{2} d x+c \int_{B^{+}(0,2 R)}\left(|D F|^{2}+|f|^{2}\right) d x .
$$


Together with Poincaré's inequality

$$
\int_{B^{+}(0,2 R)}\left|v-x_{n} \xi\right|^{2} d x \leq \frac{c}{R^{2}} \int_{B^{+}(0,2 R)}\left|D v-\xi \otimes e_{n}\right|^{2} d x
$$

this easily implies the assertion of the lemma.

Lemma A.2. Assume that $k \geq 3, A_{i j}^{\alpha \beta}, F_{i}^{\alpha} \in C^{k-1}\left(B^{+}\left(0, R_{0}\right)\right)$, and that $v \in$ $W^{1,2}\left(B^{+}\left(0, R_{0}\right)\right) \cap C^{k}\left(B\left(0, R_{0}\right)\right)$ is the solution of

$$
D_{\alpha}\left(A_{i j}^{\alpha \beta} D_{\beta} v^{j}\right)=D_{\alpha} F_{i}^{\alpha}
$$

with $v=0$ on $\partial B^{+}\left(0, R_{0}\right) \cap\left\{x_{n}=0\right\}$. Then there exists a constant $c$, which depends only on $k$ and $\left\|A_{i j}^{\alpha \beta}\right\|_{C^{k-1}\left(B^{+}\left(0, R_{0}\right)\right)}$, such that for all $0<\varrho<R<R_{0}$ (here we choose $R_{0}$ small enough so that we may apply Gärding's inequality)

$$
\begin{aligned}
& \int_{B^{+}(0, \varrho)}\left|D^{k} v\right|^{2} d x \leq c\left\{\sum_{\ell=2}^{k-1}(R-\varrho)^{-2(k-\ell)}\right. \int_{B^{+}(0, R)}\left|D^{\ell} v\right|^{2} d x \\
&\left.+\int_{B^{+}(0, R)}|D v|^{2} d x+\sum_{\ell=2}^{k-1} \int_{B^{+}(0, R)}\left|D^{\ell} F\right|^{2} d x\right\} .
\end{aligned}
$$

Proof. We argue by induction. Assume first that $k=3$. We have to show that

$$
\int_{B^{+}(0, \varrho)}\left|D^{3} v\right|^{2} d x \leq c\left\{(R-\varrho)^{-2} \int_{B^{+}(0, R)}\left|D^{2} v\right|^{2} d x+\int_{B^{+}(0, R)}|D v|^{2} d x+\int_{B^{+}(0, R)}\left|D^{2} F\right|^{2} d x\right\} .
$$

For $\sigma \in\{1, \ldots, n-1\}$ let $v_{\sigma}=D_{\sigma} v$. Then $v_{\sigma}$ is a solution of

$$
D_{\alpha}\left(A_{i j}^{\alpha \beta} D_{\beta} v_{\sigma}^{j}\right)=D_{\alpha}\left(D_{\sigma} F_{i}^{\alpha}-D_{\sigma} A_{i j}^{\alpha \beta} D_{\beta} v^{j}\right)=D_{\alpha} G_{i}^{\alpha}
$$

with $G_{i}^{\alpha}=D_{\sigma} F_{i}^{\alpha}-D_{\sigma} A_{i j}^{\alpha \beta} D_{\beta} v^{j}$ and $v_{\sigma}=0$ on $\partial B^{+}\left(0, R_{0}\right) \cap\left\{x_{n}=0\right\}$. It follows from standard regularity results in elliptic theory (see, e.g., [Gi], p. 363) that

$$
\int_{B^{+}(0, \varrho)}\left|D^{2} v_{\sigma}\right|^{2} d x \leq c\left\{(R-\varrho)^{-2} \int_{B^{+}(0, R)}\left|D v_{\sigma}\right|^{2} d x+\int_{B^{+}(0, R)}|D G|^{2} d x\right\} .
$$

By the definition of $G_{i}^{\alpha}$ and $v_{\sigma}$,

$$
\int_{B^{+}(0, \varrho)}\left|D^{2} D_{\sigma} v\right|^{2} d x \leq c\left\{(R-\varrho)^{-2} \int_{B^{+}(0, R)}\left|D^{2} v\right|^{2} d x+\int_{B^{+}(0, R)}|D v|^{2} d x+\int_{B^{+}(0, R)}\left|D^{2} F\right|^{2} d x\right\} .
$$

Rearranging the terms in the pointwise form of the differential equation, we obtain

$$
A_{i j}^{n n} D_{n n} v^{j}=D_{\alpha} F_{i}^{\alpha}-D_{\alpha} A_{i j}^{\alpha \beta} D_{\beta} v^{j}-\sum_{(\alpha, \beta) \neq(n, n)} A_{i j}^{\alpha \beta} D_{\alpha \beta} v^{j},
$$

and thus

$$
A_{i j}^{n n} D_{n n n} v^{j}=-D_{n} A_{i j}^{n n} D_{n n} v^{j}+D_{n}\left[D_{\alpha} F_{i}^{\alpha}-D_{\alpha} A_{i j}^{\alpha \beta} D_{\beta} v^{j}-\sum_{(\alpha, \beta) \neq(n, n)} A_{i j}^{\alpha \beta} D_{\alpha \beta} v^{j}\right] .
$$

It follows from the Legendre-Hadamard condition (2.4) that the matrix $\left(A_{i j}^{n n}\right)$ has a uniformly bounded inverse. Therefore we can solve the equation for $D_{n n n} v$ and 
obtain the assertion of the lemma for $k=3$, since all terms on the right hand side are estimated.

Assume now that the assertion holds for $k-1$. We want to show that the estimate (A.1) holds if $A_{i j}^{\alpha \beta}, F_{i}^{\alpha} \in C^{k-1}\left(B^{+}\left(0, R_{0}\right)\right)$. In this case $v_{\sigma}$ solves the equation (A.2) with $A_{i j}^{\alpha \beta}, F_{i}^{\alpha} \in C^{k-2}\left(B^{+}\left(0, R_{0}\right)\right)$, and we can use (A.1) for $k-1$. Thus

$$
\begin{gathered}
\int_{B^{+}(0, \varrho)}\left|D^{k-1} v_{\sigma}\right|^{2} d x \leq c\left\{\sum_{\ell=2}^{k-2}(R-\varrho)^{-2(k-\ell)} \int_{B^{+}(0, R)}\left|D^{\ell} v_{\sigma}\right|^{2} d x\right. \\
\left.+\int_{B^{+}(0, R)}\left|D v_{\sigma}\right|^{2} d x+\sum_{\ell=2}^{k-2} \int_{B^{+}(0, R)}\left|D^{\ell} F\right|^{2} d x\right\} .
\end{gathered}
$$

Thus all derivates of $v$ of order $k$ except $D_{n}^{k} v$ are estimated. Finally, we obtain the estimate for $D_{n}^{k} v$ on differentiating (A.3) $(k-2)$ times.

Lemma A.3. Assume that $\Omega$ is a domain of class $C^{k}, k \geq 2, x_{0} \in \partial \Omega$ and $2 R \leq$ $R_{0}$. Let $v \in C^{k}\left(\Omega\left(x_{0}, 2 R\right)\right)$ be a solution of

$$
D_{\alpha}\left(A_{i j}^{\alpha \beta}\left(x_{0}\right) D_{\beta} v^{j}\right)=0, \quad i=1, \ldots, m .
$$

Then for all $\xi \in \mathbb{R}^{m}$ we have

$$
\begin{aligned}
\int_{\Omega\left(x_{0}, R\right)}\left|D^{k} v\right|^{2} d x \leq & \frac{c}{R^{2(k-1)}} \int_{\Omega\left(x_{0}, 2 R\right)}\left|D v+\xi \otimes \nu\left(x_{0}\right)\right|^{2} d x+c R^{n}|\xi|^{2} \\
& +\frac{c}{R^{2(k-2)}} \int_{\Omega\left(x_{0}, 2 R\right)}|D v|^{2} d x .
\end{aligned}
$$

Proof. Assume first that $x_{0}=0$ and $\nu\left(x_{0}\right)=e_{n}$. By assumption there exists a diffeomorphism $\gamma \in C^{2}\left(B^{+}(0,2 R)\right) \rightarrow \Omega(0,2 R)$ such that $\gamma\left(B^{+}(0, R)\right)=\Omega(0, R)$ and $D \gamma(0)=\mathrm{Id}$. Let $\tilde{v}=v \circ \gamma, \Gamma_{i j}=\partial \gamma^{i} / \partial x_{j}(x)$ and $\left(\Gamma^{i j}\right)=\left(\Gamma_{i j}\right)^{-1}$. Then $\tilde{v}$ is a solution of

$$
D_{\mu}\left(\tilde{A}_{i j}^{\mu \nu} D_{\nu} v^{j}\right)=0, \quad i=1, \ldots, m
$$

with

$$
\tilde{A}_{i j}^{\mu \nu}=A_{i j}^{\alpha \beta} \Gamma^{\nu \beta} \Gamma^{\mu \alpha} \operatorname{det} D \gamma
$$

Changing coordinates, we deduce that

$$
\begin{aligned}
& \int_{\Omega\left(x_{0}, R\right)}\left|D^{k} v\right| d x \leq c \sum_{\ell=1}^{k} \int_{B^{+}(0, R)}\left|D^{\ell} \tilde{v}\right|^{2} d x \\
& \leq \frac{c}{R^{2(k-1)}} \int_{B^{+}(0,2 R)}\left|D \tilde{v}-\xi \otimes e_{n}\right|^{2} d x+c R^{n}|\xi|^{2}+\frac{c}{R^{2(k-2)}} \int_{B^{+}(0,2 R)}|D \tilde{v}|^{2} d x .
\end{aligned}
$$

The last inequality follows for $k=2$ directly from Lemma A.1; for $k \geq 3$ we apply Lemma A.2 iteratively to $D^{\ell} \tilde{v}, \ell=k, k-1, \ldots, 3$, on a sequence of half balls $B^{+}\left(0, R_{\ell}\right)$ such that $\left(R_{\ell-1}-R_{\ell}\right)^{-1} \leq c R^{-1}$, and then use Lemma A.1 to estimate 
the second derivatives. We now obtain the desired inequality in the case $x_{0}=0$, $\nu\left(x_{0}\right)=-e_{n}$, since

$$
\begin{aligned}
& \int_{B^{+}(0,2 R)}\left|D \tilde{v}-\xi \otimes e_{n}\right|^{2} d x \\
\leq & \int_{B^{+}(0,2 R)}|D v(\gamma(x))|^{2}|D \gamma(x)-D \gamma(0)|^{2} d x+\int_{B^{+}(0,2 R)}\left|D v(\gamma(x))-\xi \otimes e_{n}\right|^{2} d x \\
\leq & c R^{2} \int_{\Omega(0,2 R)}|D v|^{2} d x+\int_{\Omega(0,2 R)}\left|D v-\xi \otimes e_{n}\right|^{2} d x .
\end{aligned}
$$

In the general case we choose a rigid motion $\xi(x)=R\left(x-x_{0}\right)$ with $R \in \mathrm{SO}(n)$ such that $\tilde{\Omega}=\xi(\Omega)$ satisfies $\nu_{\tilde{\Omega}}(0)=-e_{n}$. Let $\tilde{v}=v\left(\xi^{-1}(x)\right)$. Then

$$
\begin{aligned}
\int_{\tilde{\Omega}(0,2 R)}\left|D \tilde{v}-\xi \otimes e_{n}\right|^{2} d x & =\int_{\xi\left(\Omega\left(x_{0}, 2 R\right)\right)}\left|D v\left(\xi^{-1}(x)\right) R^{t}-\xi \otimes R^{t} e_{n} R^{t}\right|^{2} d x \\
& =\int_{\Omega\left(x_{0}, 2 R\right)}\left|D v+\xi \otimes \nu\left(x_{0}\right)\right|^{2} d x .
\end{aligned}
$$

The assertion of the lemma now follows as before.

Corollary A.4. Assume that $\Omega$ is a domain of class $C^{k}, k \geq 2, x_{0} \in \partial \Omega$, and $2 R \leq R_{0}$. Let $v \in W^{1,2}\left(\Omega\left(x_{0}, 2 R\right)\right)$ be a solution of

$$
D_{\alpha}\left(A_{i j}^{\alpha \beta}\left(x_{0}\right) D_{\beta} v^{j}\right)=0, \quad i=1, \ldots, m \text {. }
$$

Then

$$
\begin{aligned}
\int_{\Omega\left(x_{0}, R\right)}\left|D^{k} v\right|^{2} d x \leq & \frac{c}{R^{2(k-1)}} \int_{\Omega\left(x_{0}, 2 R\right)}\left|D v-\left(D_{\nu\left(x_{0}\right)} v\right)_{x_{0}, R} \otimes \nu\left(x_{0}\right)\right|^{2} d x \\
& +\frac{c}{R^{2(k-2)}} \int_{\Omega\left(x_{0}, 2 R\right)}|D v|^{2} d x .
\end{aligned}
$$

Proof. Standard regularity results in elliptic theory imply that $v$ is smooth in $\Omega\left(x_{0}, 2 R\right)$. The estimate follows now from Lemma A.3 with $\xi=-\left(D_{n} v\right)_{x_{0}, R}$.

Proposition A.5. Assume that $\Omega$ is a domain of class $C^{1, \sigma}, x_{0} \in \partial \Omega$, and $v \in$ $W^{1,2}\left(\Omega\left(x_{0}, R\right)\right)$ is a solution of the elliptic system

$$
-D_{\alpha}\left(A_{i j}^{\alpha \beta}\left(x_{0}\right) D_{\beta} v^{j}\right)=0
$$

with $v=0$ on $\partial \Omega \cap \partial \Omega\left(x_{0}, R\right)$. Then

$$
\begin{gathered}
\int_{\Omega\left(x_{0}, \varrho\right)}\left|D v-\left(D_{\nu\left(x_{0}\right)} v\right)_{x_{0}, \varrho} \otimes \nu\left(x_{0}\right)\right|^{2} d x \leq c\left(\frac{\varrho}{R}\right)^{n+2} \int_{\Omega\left(x_{0}, R\right)}\left|D v-\left(D_{\nu\left(x_{0}\right)} v\right)_{x_{0}, R} \otimes \nu\left(x_{0}\right)\right|^{2} d x \\
+c R^{2 \sigma} \int_{\Omega\left(x_{0}, R\right)}|D v|^{2} d x .
\end{gathered}
$$

Proof. This is an immediate consequence of the Campanato inequality in Proposition 3.3 for the solution $\tilde{v}$ of the transformed system on $B^{+}(0, R)$. See the proof of Lemma A.3 for details. 


\section{ACKNOWLEDGMENTS}

Parts of these results are contained in my Ph.D. thesis, and it is my pleasure to thank my advisor J. Frehse for his constant support. I also thank the referee for many useful comments which helped to improve the manuscript.

\section{REFERENCES}

[AC] N. E. Aguilera, L. A. Caffarelli, Regularity results for discrete solutions of second order elliptic problems in the finite element method, Calcolo 23 (1986), 327-353. MR 89h:65171

[BLR] H. Blum, Q. Lin, R. Rannacher, Asymptotic error expansion and Richardson extrapolation for linear finite elements, Numer. Math. 49 (1986), 11-37. MR 87m:65172

[BS] S. C. Brenner, L. R. Scott, The Mathematical Theory of Finite Element Methods, Springer, New York, 1994 MR 95f:65001

[Ca1] S. Campanato, Equazioni ellittiche del $I I^{\circ}$ ordine e spazi $L^{2, \lambda}$, Ann. Mat. Pura Appl. 69 (1965), 321-381. MR 33:395

[Ca2] S. Campanato, Sistemi ellittici in forma divergenza. Regolarità all'interno, Quaderni, Scuola Normale Superiore, Pisa, 1980. MR 83i:35067

[C] P. G. Ciarlet, The Finite Element Method for Elliptic Problems, North-Holland, Amsterdam, 1978. MR 58:25001

[D] G. Dolzmann, Campanato-Ungleichungen für Differenzenverfahren und finite Elemente, Bonner Mathematische Schriften 251 (1994). MR 95h:65081

[DF] G. Dolzmann, J. Frehse, Optimale asymptotische Konvergenz linearer finite Elemente bei elliptischen Problemen mit Campanato-Abschätzungen, Bonner Mathematische Schriften 228 (1991), 95-120. MR 93j:65177

[DM] G. Dolzmann, S. Müller, Estimates for Green's matrices of elliptic systems by $L^{p}$ theory, manuscr. math. 88 (1995), 261-273. MR 96g:35054

[Du1] R. G. Durán, Quasi-optimal estimates for finite element approximations using Orlicz norms, Math. Comp. 49 (1987), 17-23. MR 88d:65160

[Du2] R. G. Durán, A note on the convergence of linear finite elements, SIAM J. Numer. Anal. 25 (1988), 1032-1036. MR 89g:65141

[F] M. Fuchs, The Green matrix for strongly elliptic systems of second order with continuous coefficients, Z. Anal. Anwendungen. 5 (1986), 507-531. MR 89a:35069

[G] M. Giaquinta, Multiple Integrals in the Calculus of Variations and Nonlinear Elliptic Systems, Princeton University Press, 1983. MR 86b:49003

[Gi] E. Giusti, Metodi diretti nel calcolo delle variazioni, Unione Matematica Italiana, Bologna, 1994.

[JN] F. John, L. Nirenberg, On functions of bounded mean oscillation, Comm. Pure Appl. Math. 14 (1961), 415-426. MR 23:A1348

[KJF] A. Kufner, O. John, S. Fučik, Function Spaces, Noordhoff, Leyden, 1977. MR 58:2189

[Na] F. Natterer, Über die punktweise Konvergenz finiter Elemente, Num. Math. 25 (1975), 67-77. MR 57:14514

[Ni] J. A. Nitsche, Schauder Estimates for Finite Element Approximations on Second Order Elliptic Boundary Value Problems, Proceedings of a Special Year in Numerical Analysis, University of Maryland, Numerical Analysis Lecture Notes 20, 1981.

[R] R. Rannacher, Zur $L^{\infty}$-Konvergenz linearer finiter Elemente beim Dirichlet-Problem, Math. Z. 149 (1976), 69-77. MR 58:8361

[RS] R. Rannacher, R. Scott, Some optimal error estimates for piecewise linear finite element approximations, Math. Comp. 38 (1982), 437-445. MR 83e:65180

[SW1] A. H. Schatz, L. B. Wahlbin, On the quasi-optimality in $L^{\infty}$ of the $\stackrel{\circ}{ }^{1,2}$-projection into finite element spaces, Math. Comp. 38 (1982), 1-22. MR 82m:65106

[SW2] A. H. Schatz, L.B. Wahlbin, Interior maximum-norm estimates for finite element methods, Part II Math. Comp. 64 (1995), 907-928. MR 95j:65143

[SZ] L. R. Scott, S. Zhang, Finite element interpolation of nonsmooth functions satisfying boundary conditions, Math. Comp. 54 (1990), 483-493. MR 90j:65021

[St] G. Stampacchia, The spaces $L^{p, \lambda}, N^{p, \lambda}$ and interpolation, Ann. Scuola Norm. Sup. Pisa (3) 25 (1965), 443-462. MR 33:7480 
[Zh] K. Zhang, A counterexample in the theory of coerciveness for elliptic systems, J. Partial Differential Equations 2 (1989), 79-82. MR 81d:35067

Max Planck Institute for Mathematics in the Sciences, Inselstr. 22-26, D-04103 Leipzig, Germany

E-mail address: georg@mis.mpg.de 